\title{
Turbulence modulation in particle-laden stationary homogeneous shear turbulence using one-dimensional turbulence
}

\author{
Marco Fistler $\odot^{*}$ \\ Chalmers University of Technology, Gothenburg 41296, Sweden
}

\author{
Alan Kerstein \\ 72 Lomitas Road, Danville, California 94526, USA
}

Scott Wunsch

Applied Physics Laboratory, Johns Hopkins University, Baltimore, Maryland 20723, USA

Michael Oevermann

Brandenburgische Technische Universität Cottbus-Senftenberg, Senftenberg 01968, Germany and Chalmers University of Technology, Gothenburg 41296, Sweden

(Received 12 June 2020; accepted 20 November 2020;

published 18 December 2020)

\begin{abstract}
Turbulence modulation in particle-laden stationary homogeneous shear turbulence (HST) is investigated using one-dimensional turbulence (ODT), a low-dimensional stochastic flow simulation model. For this purpose, an ODT formulation previously used to study turbulence modulation in forced homogeneous isotropic turbulence (HIT) is extended, so that the model emulates the anisotropic character of HST and, potentially, anisotropic turbulence in general. This is done by limiting the kinetic-energy redistribution during an eddy event to an exchange involving two velocity components, where the three possible choices of the omitted component define three eddy types whose relative likelihoods control the anisotropy. Comparisons of ODT and direct-numerical-simulation results with reference to signatures of turbulence modulation are the basis of a broader ODT parameter study that is reported. Owing to the reduced dimensionality of ODT, it is found that the fidelity of the model for single-phase HST does not extend to particle effects on flow anisotropy, but for quantities averaged over components, parametric trends are captured. The consistent approach to case comparisons that was introduced in the HIT study to evaluate sensitivities to particle-phase parameters in a given flow configuration is extended here to a cross-comparison of HST and HIT model results, and its efficacy is again confirmed. The results provide an overall characterization of the potential for ODT to support the incorporation of particle-induced turbulence modulation into subgrid-scale closures of large-eddy simulations.
\end{abstract}

DOI: 10.1103/PhysRevFluids.5.124303

\footnotetext{
*marco.fistler@gmail.com
}

Published by the American Physical Society under the terms of the Creative Commons Attribution 4.0 International license. Further distribution of this work must maintain attribution to the author(s) and the published article's title, journal citation, and DOI. Funded by Bibsam. 


\section{INTRODUCTION}

\section{A. Overview and goals}

The interaction between gas phase turbulence and a dispersed phase is ubiquitous in nature and industrial applications, and large-eddy simulation (LES) is currently the most affordable simulation tool that models particle-laden turbulent flow with adequate fidelity. For the two-way-coupling regime, which is the present focus, LESs require reliable models to capture the influence of a dispersed phase (e.g., particles or droplets) on the unresolved turbulent subgrid scales (SGSs) of the carrier phase.

These SGS models can be parametrizations whose formulations and associated parameter values should be based on accurate inputs or they can be directly data-driven. In either case, direct numerical simulation (DNS), which resolves all scales, is the preferred information source due to its fidelity and the completeness of the information that it provides [1-4]. However, the computational cost of DNS limits the parameter space that it can cover, especially for multiphysics regimes. Therefore it would be beneficial to develop an intermediate level of computational modeling that could be benchmarked against DNS data and then used to simulate the most relevant regimes for SGS closure purposes. The present study is a step toward that goal.

Before presenting the details of this strategy, it is important to characterize the phenomena, which is done next. The requirements that have to be met are discussed in Sec. IB.

The effect of the particle phase on the carrier phase turbulence is of longstanding interest and well-known under the topic of turbulence modulation by particles. In 1989, Hetsroni [5] determined based on theoretical considerations and experimental data (e.g., Refs. [6-9]) that the main parameter governing turbulence modulation is the particle Reynolds number $\operatorname{Re}_{p}$. This is given as $\operatorname{Re}_{p}=d_{p}\left|\mathbf{u}_{p}-\mathbf{u}_{f}\right| / v$, where $d_{p}$ is the particle diameter, $\left|\mathbf{u}_{p}-\mathbf{u}_{f}\right|$ is the relative velocity between particle and fluid phase and $v$ is the kinematic viscosity of the fluid phase. He supposed that the particle phase suppresses turbulence if $\operatorname{Re}_{p}$ is low, while high $\operatorname{Re}_{p}$ enhances turbulence. However, later studies by, e.g., Squires and Eaton [10], Boivin et al. [11], and Ferrante and Elghobashi [12] concluded that the ratio $\mathrm{St}=\tau_{p} / \tau_{\eta}$ of the particle response time $\tau_{p}$ and the Kolmogorov timescale $\tau_{\eta}$ has a significant effect as well, where St denotes the Stokes number. Both parameter dependences are complex for different test cases, and it cannot be conclusively determined which combinations cause suppression or enhancement on all scales. For simplicity, the following discussion will focus on monodisperse particles with a high density relative to the gas phase.

We frame our study with reference to stationary homogeneous shear turbulence (HST) because it is an especially suitable SGS representation for reasons explained in Sec. IB. Gualtieri et al. $[3,13]$ showed in their DNS study an overall suppression of turbulent kinetic energy (TKE) for this flow configuration for $\mathrm{St}=1$ and 5 . In detail for $\mathrm{St}=1$, the TKE at large turbulent length scales decreases with increasing mass loading $\phi$, but for turbulent scales smaller than the Kolmogorov length scale the TKE increases with increasing $\phi$ [3]. The same behavior is observed in studies for the related flow configuration of stationary forced, homogeneous isotropic turbulence (HIT). Boivin et al. [11] showed an overall increasing suppression effect for $\mathrm{St}=1,4,11$ with increasing $\phi$, where this trend is stronger for higher St. Again, all turbulent scales larger than the Kolmogorov length scale have decreasing TKE compared to the unladen flow.

Considering the similarity of the anisotropic characteristics of HST and wall-bounded flows, it is relevant to summarize the findings for such flows, too. Richter [14] investigated particle-laden Couette flows with DNS for bulk flow Reynolds numbers equal to 72000 and a dispersed phase with the parameters $\mathrm{St}=1-83$ and $\phi=0.25$. He showed that in the near-wall region the TKE experiences an overall damping. Additionally, the component profiles showed clearly the anisotropic characteristics, seen before in HST. While the streamwise TKE component showed almost no damping effect caused by the particle phase, the wall-normal component has one. This agrees with the HST results. Similar experimental findings by Righetti and Romano [15] support these results for a wall distance $y^{+}>100$. As in Ref. [13], this effect is weaker for higher St, but the St 
dependence is unclear. However, the investigations of wall-bounded flow support an overall damping for anisotropic particle-laden flows.

We have developed, and in this study extend, a reduced simulation tool intended to complement ongoing DNS and experimental investigation of the two-way-coupled regime of particle-laden flow. Specifically, the one-dimensional-turbulence (ODT) stochastic approach that was recently used to simulate two-way-coupled particle-laden HIT [16] is extended here for application to particle-laden HST. This extension involves an approach to ODT simulation of single-phase HST based on a modified coupling of velocity components.

ODT resolves the full range of turbulent length scales and timescales on a one-dimensional domain by solving the viscous advancement and modeling the concurrent turbulent eddy motions. Due to its reduced dimensionality, it does not capture all three-dimensional effects, but the present focus is on investigating the trends of global quantities like the fluid-phase and particle-phase TKE $k$ and $k_{p}$, respectively, and the TKE dissipation rate $\epsilon$, all per unit mass.

Previous ODT applications range from basic canonical single-phase flows [17-21] to primarybreak-up atomization [22,23] and one-way-coupled multiphase flows [24-26]. Various extensions of ODT improved its numerical capabilities and broadened its physical generality. Recently, Fistler et al. [16] formulated a model extension to two-way-coupled particle-laden, stationary, forced HIT and showed that the model features enable investigations that are complementary to DNS in terms of both the questions that can be addressed and the parameter space that can be explored affordably. On this basis, the next step toward the use of ODT to aid in the development of SGS closure of LESs is to extend ODT for application to HST, as described here.

The motivation for this study is elaborated in Sec. IB. Section II presents the ODT framework governing the fluid phase advancement. The treatment of particle-turbulence interactions is summarized in Sec. III. Sec. IV shows comparisons of ODT and DNS results and additional ODT parameter studies. The paper concludes in Sec. V with a discussion of the current state of the modeling approach and the implications of the reported results.

\section{B. Strategies for subgrid-scale closure}

SGS closure of particle-laden LESs is a motivation for the present study rather than an outcome, so the discussion of closure that follows is primarily conceptual. The main quantities that serve as LES inputs to SGS closures are the velocity difference across an LES mesh cell and the cell size or equivalent information. Dimensionally these specify both the dissipation rate in the cell and the shear across it. The dissipation rate is an energy sink at the LES level but an SGS energy source. As such, it prescribes the forcing of a notional statistically stationary HIT flow at subgrid scales. The imposed shear implies a stationary HST flow at subgrid scales if the cell size is interpreted as a periodicity scale of the flow. (This point is elaborated in Sec. II B.)

The presumed stationarity at subgrid scales reflects the shorter timescales of SGS fluid motions than of LES-resolved fluid motions. However, there is a continuum of timescales rather than a distinct timescale separation between the LES-resolved and SGS flows, so the need may arise to account for the nonstationarity of the latter. This would accentuate the need for an intermediate level of modeling that can address this affordably with sufficient fidelity.

It is assumed that the turbulence level is high enough so that resolved-flow and SGS Reynolds numbers are both high. In other words, the cell size is small enough to be in the universal range of the turbulent cascade while the cell Reynolds number is large enough so that particles that interact with the flow via two-way coupling in the notional SGS flow do so in the universal range of scales relative to the cell size. Here universality means that the phenomenology of the particle-flow interaction is insensitive to the assumed SGS flow configuration, e.g., HIT versus HST as specified above. This requires that $\mathrm{St}$ is not so large that particles interact with nonuniversal flow scales. To be clear, universality does not mean that the flow phenomenology is unique. Rather, the SGS flow has the usual dependences on, e.g., St and $\phi$, and in fact these are the dependences that the SGS closure is intended to capture. The only insensitivity is to the overall flow configuration. 
These assumptions imply turbulence levels so high that they might occur mainly in geophysical or astrophysical flows, or in the largest scale industrial processes. For the stated conditions, a continuum treatment of the dispersed phase would generally be used. Nevertheless, for multiphysics cases involving phase change, chemical reactions, etc., it is essential for the SGS closure to capture the overall effect of particle coupling to the operative microphysics, so input from numerical simulation involving Lagrangian particles remains relevant to development of the closure. In this context, a salient motivation for the use of ODT is that it can be accurately but efficiently extended to multiphysics regimes, as demonstrated in the cited references and other work. As previously in other contexts, the strategy that is envisioned is to benchmark ODT against regimes that are accessible using DNS, and then use ODT as a range extender in terms of parameter space and physical complexity.

Statistically stationary HIT is traditionally the default flow configuration for DNS in support of LES SGS closure, but it has some drawbacks, including one that is specific to two-way-coupled particle-laden flow. There is no uniquely preferable forcing, so forced HIT is not a well-defined configuration. For two-way-coupled particle-laden flow, the forcing couples to the dispersed phase in a manner that propagates its effects downscale [27]. It couples to the point-particle representation of two-way coupling in a manner that modifies flow energetics, resulting in a discrepancy between the energy dissipation rate and the nominal rate of energy production by the forcing [16].

In ODT, HIT forcing is not similarly coupled to particles, so the latter artifact is avoided, but the presence of this artifact in DNS complicates direct comparisons of DNS and ODT results [16]. This and the other noted considerations do not rule out HIT as a baseline, but they do indicate that HST is a viable alternative and perhaps preferable. In any case, for the specific purpose of studying particle-flow interaction in the universal range of scales as defined above, neither choice is precluded.

To emphasize that the outlined closure strategy does not cover all relevant regimes, we note that the scale range of the turbulence in boundary layers steadily decreases as the wall is approached, ultimately transitioning to the viscous sublayer. Therefore particle-laden boundary layers constitute a distinct class of flows that require attention, and as noted in Sec. I A, HST is relevant in this regard. There are other instances of locally moderate turbulence intensity in inhomogeneous flows, such as in free shear flows.

The research strategy that will be pursued based on these considerations is outlined in Sec. V. An aspect that is addressed in the present investigation is the level of turbulence intensity that is required in order to attain the universal regime of particle-flow-interaction phenomenology. In Sec. IV G, ODT results for HST and HIT cases that are specially configured for comparability are shown to provide guidance in this regard.

\section{FLUID PHASE}

\section{A. Modeling framework}

In this section the ODT framework governing the fluid phase advancement is described. This builds on the model described in Fistler et al. [16], to which we refer for more details. Emphasized here is the developed modeling approach for HST.

In general, ODT is a stochastic model simulating turbulent flow realizations on a one-dimensional domain. It can be seen as a representation of a line of sight in a three-dimensional flow field. For application to HST, it is oriented in the direction of the mean velocity gradient, which is denoted as the $y$ coordinate. The following description is based on the implementation of Lignell et al. [21], to which we refer readers for more details. It is a numerical method resolving the full range of timescales and length scales of the turbulent cascade along a one-dimensional line. Time advancement on this line is governed by viscous transport and turbulent advection. The latter consists of eddy events, which are a unique ODT construct that mimics line-directed turbulent stirring of fluid. Eddy events act in general as rearrangements of the velocity profiles in accordance 
with turbulence scaling laws, incorporating energy and momentum exchanges between directions and phases. Eddy events and viscous transport act alternately on property profiles, i.e., eddy events happen instantaneously between time intervals of viscous transport.

\section{B. Specialization to HST}

For HST, the ODT domain is oriented in the direction of the imposed velocity gradient. There is no mean flow in that direction, so all line-directed advection is deemed to be turbulent. Turbulent advection is represented in ODT by eddy events, so the advancement equations in Sec. II C do not include advective terms.

On this basis, the specialization to HST is accomplished primarily by the flow initialization, as specified in detail in Sec. IV B. As in DNS of HST, a jump-periodic boundary condition is applied to the streamwise velocity component, which is subject to the applied shear, while the boundary condition for the other components is periodic. With no case-specific modification of the baseline model formulation that is described in what follows, this initialization induces transient flow development. On a finite domain it is followed by a transition to statistical stationarity because the domain size constrains the largest scale of fluid motion.

This baseline model behavior is somewhat consistent with HST measurements and DNS results. It captures the distinct features of the streamwise flow relative to the other two velocity components, but it doesn't capture the structural difference between those other components. Therefore in Sec. II D, with elaboration in Sec. IIE, the ODT eddy-event formulation is extended so as to obtain a quantitative model representation of the three-dimensional anisotropic structure of HST. This shear-induced anisotropy is the distinguishing feature of HST relative to HIT.

Irrespective of this model extension, the underlying phenomenology of HST, as emulated by ODT, is turbulence production driven by the unbounded (in a notional unbounded domain) infinite reservoir of mean-flow kinetic energy that is established by the initial and boundary conditions. In this sense HST is specified by its initialization and the domain size, where the latter controls the time of occurrence of the transition to statistical stationarity and the Reynolds number of that regime. Normalization of time by the imposed shear collapses the transient development to a universal form, as elaborated in Sec. IV C.

In contrast to HST, DNS and ODT simulation of HIT require large-scale forcing not directly associated with a physical mechanism in order to maintain statistical stationarity. For this purpose, a forcing procedure was introduced in the ODT application to particle-laden HIT.

Notwithstanding these differences between HST and HIT flow characteristics and modeling, turbulent cascade phenomenology implies that the smallest scales of motion in highly turbulent flows are universal. As noted in Sec. IB, the same is expected with regard to particle-fluid coupling unless St is large enough so that particles interact with relatively large eddies. Accordingly, it is important to identify the regime of parameter space that exhibits the universality expected in the strong-turbulence limit. Owing to an artifact of the point-particle approximation it is difficult to address this directly using DNS [16], but particular features of ODT enable consistent HST-toHIT comparisons that are pertinent in this regard. Results of those comparisons are presented in Sec. IV G.

\section{Viscous advancement}

The Reynolds transport theorem in incompressible discrete form for an individual control volume with volume $V_{c}$ and with quantities stored at the cell centers yields the continuity and momentum equations

$$
\frac{d}{d t}\left(\rho V_{c}\right)=0
$$


and

$$
\frac{d u_{i}}{d t}=-\frac{1}{\rho V_{c}}\left(\tau_{i, e}-\tau_{i, w}-S_{p, i}\right) .
$$

Here the pressure term is neglected, as discussed in Kerstein et al. [18]. $u_{i}$ represents the $i$ th fluid velocity component and $\rho$ is the fluid density, which is henceforth assumed to be constant. To preserve the dimensionality of mass and momentum on the one-dimensional domain the cell volume is defined as the product of a cell cross-section area $A_{c}$ and the cell length $\Delta y$ on the ODT line. This determination can be arbitrary but will be important when treating extensive quantities, e.g., finite particle numbers. $\tau_{i}$ is the $i$ th viscous stress component evaluated on the east or west cell face and subscripted with $e$ or $w$, respectively. (The kinematic viscosity $v$ is thus introduced into the model.) $S_{p, i}$ represents the momentum exchange between particle and fluid phases that accounts for two-way coupling, as explained in Secs. III C and III E.

\section{Eddy events}

In ODT, vortical motions [28] are modeled as eddy events. An eddy event consist of two steps, a rearrangement of the flow property profiles called a triplet map and a redistribution of energy and momentum among velocity components. Additional modeling specifies the rate, locations, and sizes of eddy events [17]. A sampled eddy region is defined by its left boundary $y_{0}$ and the eddy size $l$.

The triplet map compresses each original property profile within the eddy region by a factor of three and three copies then fill the eddy region. To ensure continuity of the profiles, the second copy in the middle is spatially inverted. This process is represented mathematically as a fluid at location $f(y)$ being mapped to location $y$, where $f(y)$ is given as

$$
f(y)=y_{0}+ \begin{cases}3\left(y-y_{0}\right) & \text { if } y_{0} \leqslant y \leqslant y_{0}+\frac{1}{3} l \\ 2 l-3\left(y-y_{0}\right) & \text { if } y_{0}+\frac{1}{3} l \leqslant y \leqslant y_{0}+\frac{2}{3} l \\ 3\left(y-y_{0}\right)-2 l & \text { if } y_{0}+\frac{2}{3} l \leqslant y \leqslant y_{0}+l \\ y-y_{0} & \text { otherwise. }\end{cases}
$$

The triplet map operation conserves all quantities, increases scalar gradients, and decreases length scales, which reflects the behavior of notional turbulent eddies [25].

The directional redistribution of turbulent kinetic energy and momentum involves a kernel transformation that follows the mapping operation. The complete eddy event is denoted symbolically as

$$
u_{i}(y) \rightarrow u_{i}^{\mathrm{TM}}(y)+c_{i} K(y)+b_{i} J(y)
$$

where $u_{i}$ is the velocity in the $i$ th direction before and $u_{i}^{\mathrm{TM}}$ is the velocity after the mapping operation. For application to HST, $i=1$ is aligned with the imposed mean velocity field (streamwise direction $x$ ), $i=2$ is the mean gradient direction $y$, and $i=3$ is the spanwise direction $z$. The kernel $K(y)$ is defined as the fluid displacement profile $y-f(y)$ that is induced by the triplet map and integrates to zero over the eddy region, so, for constant density, it does not induce an overall momentum change. $J(y)$ is the absolute value of $K(y)$ and so it does not integrate to zero over the eddy region. Thus, it forces momentum change of the profiles if its coefficient $b_{i}$ is nonzero. $c_{i}$ scales the amplitude of $K(y)$.

The triplet map per se conserves momentum and energy, which leads to $b_{i}$ as

$$
b_{i}=\frac{-S_{i}}{\rho \int_{V_{e}} J d V} \equiv M_{i}
$$

and $c_{i}$ as

$$
c_{i}=\frac{1}{2 S}\left[-P_{i}+\operatorname{sgn}\left(P_{i}\right) \sqrt{P_{i}^{2}-4 S\left(T_{i}-\Delta E_{i}\right)}\right] .
$$


$S_{i}$ is the eddy momentum penalty of the $i$ th component caused by the particle-eddy interaction, explained in Sec. III E. $\Delta E_{i}$ combines redistribution of component energies and particle-fluid energy exchange. $S, P_{i}$, and $T_{i}$ are derived in Fistler et al. [16] and summarized here:

$$
\begin{gathered}
S=\frac{\rho}{2} \int_{V_{e}} K^{2} d V \\
P_{i}=\rho\left(\int_{V_{e}} u_{i}^{\mathrm{TM}} K d V+M_{i} \int_{V_{e}} K J d V\right), \\
T_{i}=M_{i} \rho\left(\int_{V_{e}} u_{i}^{\mathrm{TM}} J d V+\frac{M_{i}}{2} \int_{V_{e}} J^{2} d V\right) .
\end{gathered}
$$

$V_{e}$ is the volume of the eddy region on the ODT line.

For this redistribution, it is useful to evaluate the maximal energy $Q_{i}$ that is available to subtract. The maximum possible energy reduction (hence a negative number) $\left.\Delta E_{i}\right|_{\max }$ is the value for which the argument of the square root in Eq. (6) is zero, giving

$$
-\left.\Delta E_{i}\right|_{\max }=Q_{i}=\frac{P_{i}^{2}}{4 S}-T_{i}
$$

All components are used in HIT to redistribute the available energy, but in HST we allow only two randomly chosen components to exchange energy. This admits three different combinations $i j$. Below, $(i, j, k)$ denotes any permutation of the velocity component indices $(1,2,3)$. The probability of sampling each eddy event type is specified as

$$
p_{12}=p_{13}=\left(1-p_{23}\right) / 2,
$$

where $p_{23}$ is chosen to be 0.2 to capture the TKE proportions obtained by Rogers and Moin [29], as discussed in Sec. IV C. The physical basis of this model extension is explained in Sec. IIE.

Given the maximal extractable available energy, $Q_{i}$, the energy change for a given component is specified as

$$
\Delta E_{i}=\alpha\left[Q_{j}-Q_{i}\right]-S_{E, i}
$$

$S_{E, i}$ represents the sum over particles of interphase energy penalties and is defined in Sec. IIIE. $\alpha$ is an additional model parameter with its allowed range $0 \leqslant \alpha \leqslant 1$ and determines the extent of the redistribution of energy among the velocity components. $\alpha$ is chosen to be 1 , similar to Ashurst and Kerstein [19]. For the nonparticipating component $k, \Delta E_{k}=-S_{E, k}$.

The rate of eddy occurrences depends on the current flow state within any given interval $\left[y_{0}, y_{0}+l\right]$. This evaluation differs for HST only in the determination of the eddy timescale $\tau_{e}$. To determine the eddy timescale $\tau_{e}$ we use the available kinetic energy in a sampled size- $l$ region only for components $i$ and $j$. The reason is that individual eddy events in HST are idealized as two-dimensional motions, as explained in Sec. II E. Based on the scaling assumption for kinetic energy $E_{\text {kin }} \sim \frac{1}{2} m \sum_{i} u_{i}^{2} \sim \frac{1}{2} \rho V_{e} \frac{l^{2}}{\tau_{e}^{2}}$, the eddy timescale is modeled as

$$
\frac{1}{\tau_{e}}=C \sqrt{\frac{2}{\rho V_{e} l^{2}}\left(E_{\mathrm{kin}}-Z E_{\mathrm{vp}}\right)},
$$

where $E_{\text {kin }}=\frac{K K}{V_{e} l^{2}}\left(Q_{i}+Q_{j}-S_{E, i}-S_{E, j}\right)$ and $K K=\int_{V_{e}} K^{2} d V$. The viscous penalty energy is given as $E_{\mathrm{vp}}=\frac{V_{e}}{2 l^{2}} \frac{\mu^{2}}{\rho}$ and $\rho$ and $\mu$ are eddy volume averages of density and dynamic viscosity, respectively. In this study, they are constant. $C$ is an adjustable parameter that scales the overall eddy event frequency. $Z$ is the viscous penalty parameter, which suppresses nonphysical small eddies. 


\section{E. Anisotropic eddy events}

ODT is an anisotropic construction because the spatial structure of the flow is captured only along the coordinate $y$ aligned with the ODT domain. This distinguishes the lateral $(y)$ direction, which is aligned with the mean velocity gradient in the application to HST, from the streamwise $(x)$ and spanwise $(z)$ directions. As explained in Sec. II B, the specific anisotropic features of application cases have previously been represented in ODT only through the influence of the initial and boundary conditions and forcings on the modeled advancement processes. The typical outcome is that the velocity component that is subject to TKE production, usually the streamwise component, is distinguished from the other two components, which are statistically equivalent. For HST this yields a normalized streamwise velocity variance that exceeds the other normalized component variances, where the latter are equal. This is a partial representation of HST anisotropy.

The physical process that produces HST anisotropic structure is the anisotropic TKE production forced by the spatially uniform shear, balanced by the tendency of turbulent eddy motions to restore isotropy. The initial condition implies spatially uniform $z$-directed vorticity whose principal instability is generation of motion in the $x-y$ plane that is a direct source of streamwise $\left(u_{1}\right)$ variance through the production term in the TKE budget, some of which is transferred to the lateral $\left(u_{2}\right)$ variance through pressure scrambling. Secondary instabilities generate fully three-dimensional motions, with the net outcome $\left\langle u_{1}^{\prime 2}\right\rangle>\left\langle u_{3}^{\prime 2}\right\rangle>\left\langle u_{2}^{\prime 2}\right\rangle$, as seen in Table I.

To obtain more precise conformance to HST flow structure for the purpose of studying fluidparticle interaction in HST, a specialization of ODT to HST is formulated that yields rough agreement with the HST partitioning of TKE among the three components. Elaborating on the notion that an instability of the imposed spanwise vorticity can be viewed as generating twodimensional motion normal to $z$, each eddy event is viewed as a two-dimensional motion normal to one of the three coordinate directions. This implies that intercomponent energy exchange should be applied only between the two components in the plane of motion, as indicated by Eq. (12). Hence there are three eddy types, where the probability of sampling each type is adjusted as described in Sec. II D in order to obtain rough agreement with the anisotropic structure indicated by the DNS results in Table I.

Importantly, a sampled eddy event is typically not implemented because eddy events are oversampled and then culled by comparing the sampling rate to the rate implied by Eq. (13), as explained in the cited references. $E_{\text {kin }}$ in Eq. (13) is in general different for each eddy type for a given instantaneous flow state in the eddy interval, and the differences can have systematic tendencies for an anisotropic configuration such as HST. Therefore the relative occurrence rates of the three eddy types does not in general conform to the sampling probabilities assigned using Eq. (11). However, if the flow configuration is isotropic, as in the cited HIT study, and each eddy type is assigned sampling probability $1 / 3$, then there is no symmetry-breaking mechanism so the occurrence rates of the three eddy types are equal and the simulated flow is isotropic, although not statistically equivalent to HIT simulated using one eddy type.

More broadly, the use of three eddy types with equal sampling probabilities is a valid generalpurpose alternative to ODT with one eddy type. A potential advantage is that the unphysical statistical equivalence of two of the velocity components that occurs for various shear-flow configurations can then be broken by adjusting the probabilities without reformulating the model.

\section{PARTICLE PHASE}

\section{A. Elements of the particle treatment}

The particle phase treatment description that follows is a short summary of the work of Schmidt et al. [24], Sun et al. [25,26], and Fistler et al. [16]. Analogously to the fluid-phase treatment, it consists of time advancement punctuated by interactions with intermittently occurring eddy events, which we call particle-eddy interactions (PEIs) in the following. Both are governed by the drag law (Sec. III B). Due to their different implementations, they are described separately in Secs. III C 
and III D. They both are formulated to enforce the physical limits of no-slip ( $\left.\tau_{p} \rightarrow 0\right)$ and ballistic $\left(\tau_{p} \rightarrow \infty\right)$ particle behavior.

\section{B. Drag law}

As in the DNS comparison cases, we use the point-source approximation due to the assumption of relatively small particle sizes compared to the Kolmogorov length scale of the fluid phase. Additionally, the particles are assumed to be spherical [30] and reasonably modeled in a Lagrangian way following Newton's second law of motion [31]. For low particle Reynolds number and high density ratio, the governing equations for each individual particle, here omitting gravity and adopting the point-particle approximation, are

$$
\begin{aligned}
\frac{d v_{p, i}}{d t} & =-\frac{v_{p, i}-v_{f, i}}{\tau_{p}} f, \\
\frac{d x_{p, i}}{d t} & =v_{p, i} .
\end{aligned}
$$

The subscripts $p$ and $f$ represent the particle and the fluid phase, respectively, and the coordinate $i=$ 2 is aligned with the ODT coordinate direction $y$ as in Sec. II. Here $v_{f, i}$ represents the undisturbed fluid velocity as specified in Sec. III C. $x_{p, 2}$ is the particle spatial coordinate on the ODT line and $v$ is used instead of $u$ to denote velocities because the velocities used to advance these equations are not necessarily the same as for the ODT fluid-phase velocities $u_{i}$.

The response time, $\tau_{p}=\left(\rho_{p} / \rho_{f}\right)\left(d_{p}^{2} / 18 v\right)$ based on Stokes flow, is expressed here in terms of the diameter $d_{p}$, the particle-to-fluid density ratio $\rho_{p} / \rho_{f}$, and the fluid kinematic viscosity $\nu$. (Where convenient, the dynamic viscosity $\mu=\rho_{f} v$ is introduced.) An empirical correction factor $f$ is specified in terms of $\operatorname{Re}_{p}$, defined in Sec. I A, as

$$
f=1+0.15 \operatorname{Re}_{p}^{0.687}
$$

based on studies of Schiller and Naumann [32] for nonslip Reynolds numbers $\operatorname{Re}_{p}$ smaller than 200 (incorrectly stated to be 800 in Ref. [16]) to capture the standard drag curve [30]. This correction is not used in the DNS comparison cases, but it is included here for consistent comparison to previously reported HIT results. Here $\mathrm{Re}_{p}$ is evaluated with the interacting gas velocities, which differ for particle time advancement and particle-eddy interaction, as explained in what follows. The drag law [Eq. (14)] is solved by a first-order Euler method.

\section{Particle time advancement}

An essential assumption of ODT is that the advection of fluid elements occurs only by triplet mapping and thus the velocity components $v_{f, i}$ in Eq. (14) cannot directly be set equal to the ODT fluid velocities $u_{i}$. Considering the zero-inertia limit of vanishing $\tau_{p}$, particles cannot deviate from the trajectory of the fluid elements that contain them, and so $v_{p, 2}$ is forced to the value zero. The only value of $v_{f, 2}$ in Eq. (14) that is consistent with this requirement is zero. In the other coordinate directions $v_{f, i}$ is taken to be $u_{i}$, evaluated at the current particle location $x_{p, 2}$ on the ODT domain, for those components, because they have no bearing on the displacement of particles relative to fluid elements along the ODT domain. $u_{i}$ is concurrently time advanced using Eq. (2), in which the term $S_{p, i}$ provides the back-coupling from the particle to the fluid. As in conventional point-particle numerical implementation, the coupling distributes the particle-induced momentum change of the fluid uniformly within the mesh cell containing the particle.

The other limiting case of infinite-inertia ballistic particles is covered by Eq. (14), which gives constant-velocity motion for any time history of the fluid velocity components in this limit. Therefore, this limiting case requires no extra treatment.

During the integration of Eq. (14) the particle momentum changes. To maintain momentum conservation the equal and opposite momentum change is applied to the fluid within the mesh cell 
that contains it. The particle feedback to the fluid phase in Eq. (2) is therefore

$$
S_{p, i}=\sum_{N_{c}} m_{p}\left(v_{p, i}-v_{f, i}\right) \frac{f}{\tau_{p}},
$$

where $N_{c}$ is the number of particles in the mesh cell (but particle and mesh cell indices are omitted in the summand for clarity). The determination of the particle mass $m_{p}$ from the inputs that define each case is explained in Sec. IV D.

\section{Particle-eddy interaction}

The treatment of the particle phase during its interaction with an instantaneous eddy event is termed the particle-eddy interaction (PEI) in Sec. III A. This treatment is governed by the geometrical and mathematical characteristics of an eddy event, described briefly in Sec. II D, and need to be distiguished from the physical vortex/eddy structures seen in experiments and DNS. Introduced by Schmidt et al. [24] and noted as type-I, the one-way coupling approach prescribes instantaneous domain-aligned displacements of particles located in the sampled eddy region of an eddy event. The PEI uses an eddy lifetime defined as the eddy timescale $\tau_{e}$ [Eq. (13)] times a coefficient $\beta_{p}$, which is a model parameter, that is the maximum interaction time duration. It can also be shorter in duration if the particle exits the volume occupied by the eddy, defined below, before the end of the eddy lifetime.

A notional $[l \times l \times l]$ volume in which the PEI is deemed to happen defines a so-called eddy box spanned by the sampled eddy interval $l$ and intervals $[-l / 2, l / 2]$ in each of the two coordinate directions normal to the ODT line direction. This defines a notional cross section, intersecting the ODT line at $[0,0]$, that is eddy-specific owing to the range of possible $l$ values and therefore is not the same as the spatially uniform time invariant cross section $A_{c}$ defined in Sec. II A that is used to relate intensive and extensive quantities. This means the PEI is terminated either because the eddy motion has ended or because the particle leaves the volume containing the eddy before the end of the eddy motion. Although the model lives on the one-dimensional domain, the PEI evolves all three components of fluid and particle velocity and therefore can evolve the particle trajectory in three dimensions under simplifying assumptions such as constancy of domain-normal fluid velocity components in the domain-normal directions.

To determine the trajectory of a particle in the eddy box and its box exit time, modifications of the drag law are needed in order to satisfy consistency conditions. Under the assumption that $v_{f, i}$ and $\tau_{p} / f$ are constant in time during the PEI and are evaluated at the initial particle location, the drag law can be simplified and integrated to obtain an analytical solution over an arbitrary time interval $t$,

$$
v_{p, i}=v_{f, i}-\left(v_{f, i}-v_{p 0, i}\right) e^{-t f / \tau_{p}}
$$

This yields the particle trajectory

$$
x_{p, i}=x_{p 0, i}+v_{f, i} t-\frac{\tau_{p}}{f}\left(v_{f, i}-v_{p 0, i}\right)\left(1-e^{-t f / \tau_{p}}\right) .
$$

For $i \neq 2, v_{f, i}$ is chosen to be the value of $u_{i}$ at the particle initial location $y_{p 0} \cdot v_{f, 2}$ is defined differently for consistency with the two limits of zero-inertia and ballistic particles and will be explained shortly. The auxiliary variables $x_{p, i}$ for $i \neq 2$ are initialized to nominal values $x_{p 0, i}=0$. The exit times in $i \neq 2$ directions are governed by the particle trajectory given by Eq. (18). As noted, $i=2$ requires a modified treatment that is given below. Nevertheless, the first crossing of any face of the eddy box determines the exit time.

The triplet map displaces fluid elements within the eddy region. Zero-inertia particles must experience the same displacements as fluid elements during the PEI, which requires the interacting velocity $v_{f, 2}$ to be defined as the quotient of displacement over eddy lifetime. Although the triplet map provides three possible massless particle positions, a unique position is sampled randomly with 
equal likelihoods from those three possible ones. The mathematical justification for this is presented in the Appendix of Fistler et al. [16].

At the other limit, the PEI should have no effect on a ballistic particle as its response time $\tau_{p}$ goes to infinity. Using Eqs. (17) and (18) to determine the exit position and velocity would violate this, motivating the adoption of the modified PEI formulation

$$
\begin{aligned}
\Delta y_{p} & =v_{f, 2} t_{\mathrm{pei}}-v_{f, 2} \frac{\tau_{p}}{f}\left(1-e^{-t_{\mathrm{pei}} f / \tau_{p}}\right), \\
\Delta v_{p, 2} & =v_{f, 2}\left(1-e^{-t_{\mathrm{pei}} f / \tau_{p}}\right),
\end{aligned}
$$

which conserves both limits. The detailed reasoning that yields this modification based on enforcement of the physically correct no-slip and ballistic limiting behaviors is provided in the previously mentioned studies. The $\Delta y_{p}$ equation corrects the omission of the denominator in Eq. (29) of Ref. [16].

Equation (19) differs from a physically consistent drag-law formulation for finite St, but smoothly interpolates between the limits in which it is physically correct. From this viewpoint the approximation is no more severe than idealizations inherent in the overall modeling framework.

\section{E. Interphase coupling terms}

The momentum and energy exchange in direction $i=2$ during the PEI requires a coupling back to the eddy events in Sec. IID akin to the treatment of particle time advancement in Sec. III C. $S_{i}$ and $S_{E, i}$ in Eqs. (5) and (12), respectively, enforce fluid momentum and energy changes that are equal and opposite to those of the particle phase, where

$$
S_{i}=\sum_{N_{\mathrm{e}}} m_{p} \Delta\left(v_{p, i}\right)
$$

and

$$
S_{E, i}=\frac{1}{2} \sum_{N_{\mathrm{e}}} m_{p} \Delta\left(v_{p, i}^{2}\right) .
$$

These are summations over the $N_{\mathrm{e}}$ particles within the eddy volume, with the summation indices suppressed in the summands.

\section{RESULTS}

\section{A. Scope of the investigation}

In this section, the results of single-phase and particle-laden ODT are presented. Single-phase results are reported for transient and stationary regimes and are validated against data of Rogers and Moin [29] and Gualtieri et al. [3], respectively. The particle-laden results are compared with the observations of Gualtieri et al., allowing adjustment of the model parameter $\beta_{p}$. The following part presents ODT predictions for a wide parameter range of $\mathrm{Re}_{\lambda}$, St, and $\phi$, including the DNS data of Battista et al. [13]. Additionally, stationary forced HIT cases using the three-eddy-type submodel are used to evaluate the case sensitivity of turbulence modulation effects.

\section{B. Single-phase setup}

The single-phase ODT setup is described here and the incorporation of particles is explained in Sec. IV D. The initial condition is uniform shear $S=\partial u_{1} / \partial y=0.5 \mathrm{~s}^{-1}$ in the ODT line direction over the domain and $u_{i \neq 1}(y)=0$. The ODT domain length $D$ is set to $D=14 \mathrm{~m}$ with periodic boundary conditions (jump-periodic for $u_{1}$ ). Based on parameter settings that produce results in conformance with the DNS comparison case for the statistically stationary regime (Table II), $D$ is roughly 10 times the integral length scale $L_{11}$, which is defined in Eq. (23). Eddy event sizes are 
TABLE I. Transient statistics in HST using DNS [29] and ODT at $S \times t=8.0$.

\begin{tabular}{llcc}
\hline \hline & & \multicolumn{2}{c}{ ODT } \\
\cline { 3 - 4 } & DNS & $C=4$ & $C=6$ \\
\hline$\left\langle u_{1}^{\prime 2}\right\rangle / k$ & 1.06 & 0.98 & 0.98 \\
$\left\langle u_{2}^{\prime 2}\right\rangle / k$ & 0.32 & 0.30 & 0.35 \\
$\left\langle u_{3}^{\prime 2}\right\rangle / k$ & 0.62 & 0.72 & 0.69 \\
$-\left\langle u_{1}^{\prime} u_{2}^{\prime}\right\rangle / k$ & 0.33 & 0.32 & 0.51 \\
$P / \epsilon$ & 1.4 & 1.18 & 1.22 \\
$S k / \epsilon$ & 4.30 & 3.68 & 2.38 \\
\hline \hline
\end{tabular}

limited to a maximum of $70 \%$ of the domain size. This bound is necessary to capture the low-wavenumber shape of the DNS TKE spectrum. Here the same value of the viscous penalty parameter [Eq. (13)] is used as in Ref. [16], so $Z=600$. The sensitivity of the transient statistics to the eddy event frequency parameter $C$ is evaluated in Sec. IV C.

The transient ODT results are the ensemble average of 1024 realizations. The stationary results are obtained by starting from fully developed turbulent profiles and averaged over $\sim 5000$ times the integral timescale. ODT is solved on an adaptive mesh, but to gather the statistics of the rate of dissipation of TKE during the simulation, the current system state is duplicated, with interpolation, on a uniform grid with 500 grid cells that is used for further data reduction. Additionally, the simulation creates output files of the current state at uniform time intervals. These are used to obtain the kinetic-energy spectrum.

\section{Single-phase results}

The only parameter defining HST in an infinite domain is the uniform shear $S$. If this is used to normalize time and length is normalized by $\sqrt{v / S}$, then the flow evolution is parameter-free and hence universal. As the turbulence intensity increases during the flow development, the initial condition relaxes to self-similar transient evolution during which ratios of quantities controlled by the large-scale motions are time invariant. In a physical or numerically simulated realizations of HST, the finite domain bounds the scales of motion, resulting in a transition to statistically stationary time advancement. Large-scale structure is then influenced by the jump-periodic boundary condition applied to $u_{1}(y)$, which can have a leading-order effect on large-scale properties. Nevertheless, a set of ODT parameters is identified that for present purposes is sufficiently accurate in both regimes.

Table I compares DNS [29] and ODT statistics of the transient flow at the nondimensionalized time $S \times t=8$.0. Owing to the self-similar evolution at this normalized time, its specific value is immaterial. $k$ denotes the TKE level, $\epsilon$ is the rate of TKE dissipation. Here results for eddy event frequency parameter values $C=4$ and 6 are compared. $C$ mainly affects the shear stress $-\left\langle u_{1}^{\prime} u_{2}^{\prime}\right\rangle$, which directly determines the production term of TKE given as $P=-\left\langle u_{1}^{\prime} u_{2}^{\prime}\right\rangle S$. Due to the fact that $C$ governs the frequency of eddy events [Eq. (13)] and that the eddy events are the only mechanism that advects velocity fluctuations along the domain, increasing $C$ directly increases $P$. Consequentially, the TKE level reaches saturation more rapidly for higher $C$.

Overall, the ODT data show good agreement with the DNS. The different $C$ values show different prediction accuracies. $C=4$ captures better $-\left\langle u_{1}^{\prime} u_{2}^{\prime}\right\rangle / k$ and $S k / \epsilon$. The ratio between production $P$ and dissipation $\epsilon$ is slightly better matched for $C=6$. In summary, each parameter value has different prediction strength and it is up to the user to define the most important characteristic.

However, considering the individual magnitudes of $k$ and $\epsilon$ for the stationary test case from direct numerical simulations (DNS) studied by Gualtieri et al. [3] the ODT results for $C=6$ show the most accurate prediction (see Table II). Therefore, $C=6$ is used for all stationary cases. Using ODT, three single-phase test cases were carried out with Taylor microscale Reynolds number 
TABLE II. Turbulence characteristics of all single-phase test cases of DNS [3] and ODT.

\begin{tabular}{lcclccrcc}
\hline \hline & Inputs & Outputs & & & & & & \\
\hline & $v\left[\frac{\mathrm{m}^{2}}{\mathrm{~s}}\right]$ & $k\left[\frac{\mathrm{m}^{2}}{\mathrm{~s}^{2}}\right]$ & $\epsilon\left[\frac{\mathrm{m}^{2}}{\mathrm{~s}^{3}}\right]$ & $\eta[\mathrm{m}]$ & $\tau_{\eta}[\mathrm{s}]$ & $\mathrm{Re}_{\lambda}$ & $L_{11}[\mathrm{~m}]$ & $T_{11}[\mathrm{~s}]$ \\
\hline DNS & 0.0125 & 0.556 & 0.088 & 0.070 & 0.377 & 43 & 1.56 & 2.56 \\
ODT & 0.0125 & 0.523 & 0.086 & 0.069 & 0.380 & 41 & 1.356 & 2.814 \\
ODT & 0.0010 & 0.455 & 0.0597 & 0.011 & 0.129 & 152 & 1.180 & 2.624 \\
ODT & 0.0005 & 0.440 & 0.0356 & 0.0077 & 0.119 & 269 & 1.205 & 2.726 \\
\hline \hline
\end{tabular}

$\operatorname{Re}_{\lambda}=2 k \sqrt{5 /(3 v \epsilon)}$ values of 43,152, and 269. $v$ is the kinematic viscosity and for $k$ and $\epsilon$ the stationary values are used. The turbulence characteristics of DNS and ODT are summarized in Table II including macro- and micro- timescales and length scales. The microscales $\tau_{\eta}$ and $\eta$ and the Taylor microscale $\lambda$ are based on combinations of $\nu, k$, and $\epsilon$ as follows:

$$
\tau_{\eta}=\sqrt{v / \epsilon}, \quad \eta=\epsilon^{-\frac{1}{4}} \nu^{\frac{3}{4}}, \quad \lambda=\sqrt{10 \nu k / \epsilon} .
$$

The value for the TKE dissipation rate $\epsilon$ in ODT is implicitly set through the choice of eddy frequency parameter $C$ [Eq. (13)] to match the DNS $\epsilon$ value and thus the inferred quantities $\operatorname{Re}_{\lambda}$ and $\lambda$. The integral length scale and timescale, $L_{11}$ and $T_{11}$, are given as

$$
L_{11}=\frac{\pi}{2 u^{\prime 2}} \int_{0}^{\kappa_{\max }} \frac{E(\kappa)}{\kappa} d \kappa, \quad T_{11}=\frac{L_{11}}{u^{\prime}}
$$

where $E(\kappa)$ is the kinetic energy spectrum. $u^{\prime}$ is the average fluctuation given as $u^{\prime}=\sqrt{\frac{2}{3} k}$. $L_{11}$ and $T_{11}$ are predicted in contrast to the outputs that precede them in Table II.

The nondimensionalized TKE spectra in Fig. 1 approach the inertial-subrange slope $-5 / 3$ for increasing $\operatorname{Re}_{\lambda}$. In the viscous range ODT spectra tend to collapse to a shallower slope than the DNS data owing to the high-wave-number content of the discontinuous velocity derivatives introduced by the triplet map [17]. This has also been seen to a lesser degree in HIT TKE spectra [16]. It does not have a large impact on global turbulence properties, but its influence on particle-flow interactions is noted in Sec. IV E.

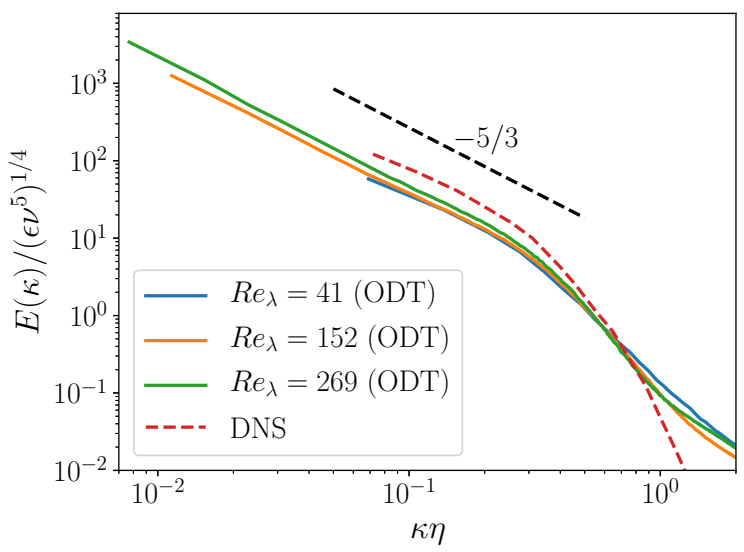

FIG. 1. Nondimensionalized kinetic-energy spectrum of the fluid phase. 
TABLE III. Mean particle number per volume $\eta^{3}$ for mass loading $\phi=1.0$. (Multiply by $\phi$ to obtain $n_{p} \eta^{3}$ for other mass loadings.)

\begin{tabular}{lcc}
\hline \hline & $\operatorname{Re}_{\lambda}=43$ & $\operatorname{Re}_{\lambda}=84$ \\
\hline $\mathrm{St}=1$ & 1.41 & 0.77 \\
$\mathrm{St}=5$ & 0.10 & 0.06 \\
\hline \hline
\end{tabular}

\section{Setup for particle-laden flow}

A particle-laden simulation is initialized by first running a single-phase case for the desired value of $\operatorname{Re}_{\lambda}$ until it reaches statistical stationarity. Then particles are randomly distributed along the ODT line. The initial particle velocities are assigned by interpolating the discretized fluid velocity profile, thus starting with zero slip velocity. In like manner as the fluid phase, the particle displacements and velocities obey periodic boundary conditions. $v_{p, 1}$ in particular has jump-periodic boundary conditions, which add or subtract $S \times D$ after exiting the domain from left or right, respectively. After particles are introduced into the equilibrated single-phase ODT state, the flow is time advanced for sufficient additional time for the two-phase flow to reach statistical stationarity before the gathering of output statistics begins.

The particle phase is nominally defined for a two-way coupling simulation by the three quantities Stokes number St, density ratio $\frac{\rho_{p}}{\rho_{f}}$, and mass loading $\phi$. For one-way coupling between particle and fluid phase the latter quantity is not required. Using these three quantities and fluid properties in Table II, and assuming that particles are spherical, the particle relaxation time $\tau_{p}$, the particle diameter $d_{p}$, the mass of a single particle $m_{p}$, and the particle number density $n_{p}$ can be calculated by

$$
\begin{aligned}
\tau_{p} & =\operatorname{St} \tau_{\eta}, \quad d_{p}=\sqrt{18 v \operatorname{St} \tau_{\eta} \frac{\rho_{f}}{\rho_{p}}} \\
m_{p} & =9 \pi \sqrt{\frac{2}{\rho_{p}}\left(\operatorname{St} \tau_{\eta} v \rho_{f}\right)^{3},} \quad n_{p}=\frac{\phi \rho_{f}}{m_{p}} .
\end{aligned}
$$

$\rho_{f}$ is assigned the nominal value $1.0 \mathrm{~kg} / \mathrm{m}^{3}$, allowing the evaluation of $m_{p}$ and $n_{p}$. The expression for $n_{p}$ is the relation that defines $\phi$. Equation (34) in Ref. [16] wrongly expresses $n_{p}$ by including $L$, which needs to be omitted as in Eq. (24). $n_{p}$ normalized by $\eta^{3}$ or $L_{11}^{3}$ is generally deemed to have negligible influence on the global quantities considered here for given values of the case-defining parameters. As a practical matter there is insufficient comparison data for validation of any such influence that ODT might identify, so this is not addressed here. It could however have significant influence on important fluctuation properties such as the probability density function of the energy dissipation rate, so this will be examined in future work, but $n_{p} \eta^{3}$ values are recorded in Table III for all ODT cases presented in Sec. IV F.

Noteworthy is that each quantity in Eq. (24) depends on some power of the ratio of fluid and particle density. Due to the DNS data compared here, this study uses a fixed value $\rho_{p} / \rho_{f}=800$, but additional examination is required in the future to study its effect.

Consistent with the DNS results, St is not reevaluated for case-specific values of $\tau_{\eta}$. Instead, the single-phase value of $\tau_{\eta}$ is used in order to simplify the interpretation in the following sections. It was shown in Ref. [16] that physically more intuitive parameter dependences are generally obtained when parameter values are based on the case-specific flow state rather than a nominal reference state, so future applications of ODT to particle-laden HST will use case-specific parameter definitions.

All cases are monodisperse because the available DNS comparison cases are monodisperse. However, the model straightforwardly generalizes to polydispersions. All presented cases result in 


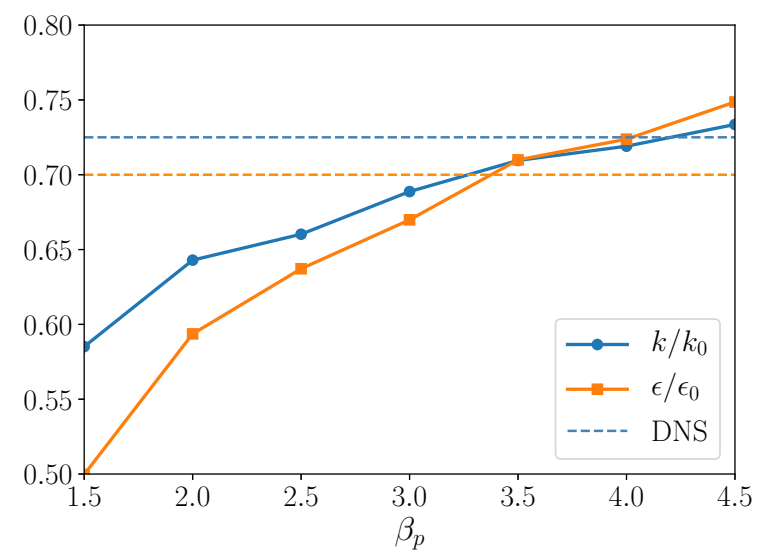

FIG. 2. Parameter study of $\beta_{p}$ to fit the fluid-phase TKE $k$ and its dissipation rate $\epsilon$ to DNS [3] results for $\operatorname{Re}_{\lambda}=43, \mathrm{St}=1$, and $\phi=0.8$.

particle volume loadings which are in the two-way coupling regime without significant collision effects (which are therefore neglected here) [33]. As mentioned before, ODT is solved on a nonuniform adaptive mesh with a minimum size of 0.001 of the domain length which gives $d_{p}(\mathrm{St}=$ $1) / \Delta_{\min }=0.25$ and $d_{p}(\mathrm{St}=5) / \Delta_{\min }=0.58$. However, the simulation almost never reached that point.

\section{E. Turbulence modulation for $\operatorname{Re}_{\lambda}=43$}

Completion of the ODT setup for particle-laden flow requires evaluation of the particle-eddyinteraction parameter $\beta_{p}$. Figure 2 shows a parameter study based on the turbulent kinetic energy $k$ and the dissipation rate $\epsilon$ for $\mathrm{St}=1$ and $\phi=0.8$. It is seen that the sensitivity of $k$ and $\epsilon$ to $\beta_{p}$ is significant. In this study, $\beta_{p}$ is set to 3.5, which agrees the best with the DNS values of $k$ and $\epsilon$ for this case according to Fig. 2.

In Fistler et al. [16], $\beta_{p}$ is assigned value 0.8 based on comparison to DNS particle dispersion statistics for HIT, which are not available for HST. This highlights the need for DNS outputs that would enable consistent model benchmarking across flow configurations. A DNS output statistic that could be the basis of a potentially robust determination of $\beta_{p}$ is proposed in Sec. V.

Figure 3 shows the effect of the particle phase on the nondimensionalized TKE spectra. As the matching global TKE levels over the range of mass loadings suggests, the integrals of the spectra agree well with the DNS results. It is instructive to compare Fig. 3(b) to Fig. 4 of Ref. [16], in which DNS and ODT spectra for particle-laden HIT for several St values are shown and the associated mechanisms are explained. For both flows, the following are seen: (i) each DNS and ODT spectrum ratio has a local minimum, (ii) the best agreement of ODT with DNS is at low wave numbers, (iii) at high wave numbers, DNS shows strong spectrum enhancement relative to the unladen case but ODT does not. In Ref. [16] it was noted that DNS and ODT are subject to different forms of numerical dissipation associated with particle-fluid coupling, making the interpretation of this discrepancy unclear.

The comparison of the HST and HIT spectra is clearest for the one case shown in both figures, namely, $\mathrm{St}=1, \phi=0.4$. For this case, both ODT and DNS indicate that the minimum of the spectrum ratio is at $\kappa \eta=0.4$ for both flows, and that the minimum is lower for HIT than for HST. However, for ODT the HIT minimum is lower than the DNS value, but the ODT HST minimum is higher than the DNS value, so ODT indicates a greater difference between the HIT and HST minima than does DNS. 


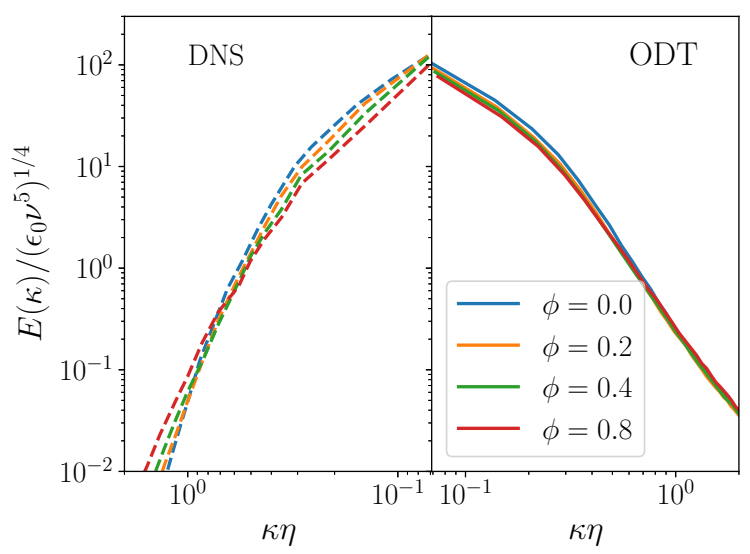

(a)

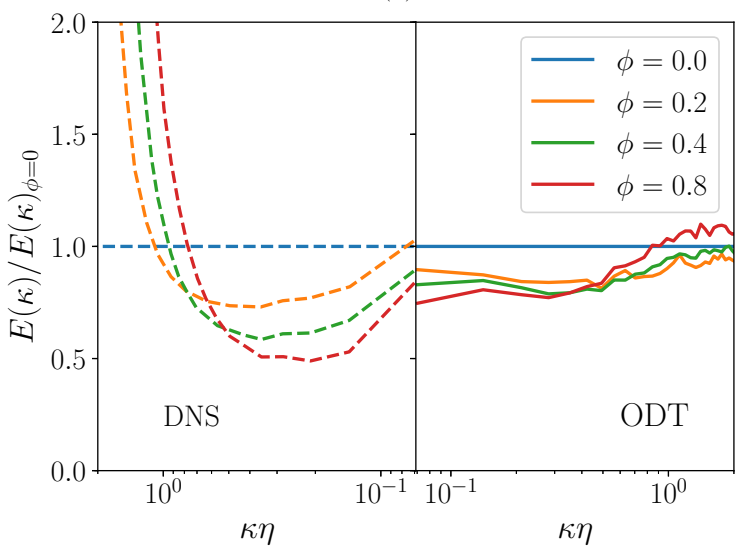

(b)

FIG. 3. Dependence of the nondimensionalized spectrum on mass loading. $\operatorname{Re}_{\lambda}=43, \mathrm{St}=1$ (DNS by Gualtieri et al. [3]). $\epsilon_{0}$ is the dissipation rate for the unladen case.

Overall, ODT is in closer agreement with DNS for HIT than for HST. One reason is the model artifact noted in Sec. IV C, namely, the ODT high-wave-number spectral falloff is too slow owing to spurious discontinuous velocity derivatives caused by triplet maps. This tends to dominate the spectral signature of the transfer of kinetic energy to high wave numbers by particle-flow interactions, thereby suppressing the sensitivity of the spectra to the latter in ODT. The effect is more pronounced for HST owing to the imposed velocity gradient, which results in the mean flow as well as velocity fluctuations contributing to the severity of the map-induced derivative discontinuities.

Beyond this, the discrepancy reflects an artifact that is specific to the ODT representation of twoway coupling. Theory and numerical simulation of the two-way-coupling regime [27,34] identify a wave-number range in the vicinity of the Kolmogorov wave number in which this coupling boosts the spectral amplitude, as reflected in the DNS results in Fig. 3(b). In this wave-number range, ODT flow evolution is dominated by viscous advancement, governed by Eq. (2). That equation has no advective term because the eddy event is the sole advection process in the present model formulation. Due to this truncation of the equation, ODT does not fully capture the mechanism by which the particle-fluid interaction boosts dissipation-range fluctuations, so the effect is weaker than for DNS, although the dependence on $\phi$ is qualitatively captured.

Several considerations complicate the interpretation of the results. One is that parameter adjustment was based on different criteria in the two model applications. Another is that the HIT 
comparison of ODT and DNS involved St variation, but here it involves $\phi$ variation. However, a $\phi$ sensitivity of ODT for fixed St, in the format of Fig. 3(b), was also shown (Fig. 8 of Ref. [16]). There was no comparison of the results to DNS because the dissipation rate was held fixed at the unladen value, a setup for which there are no DNS data. There is little difference between the ODT results for $\mathrm{St}=1$ and $\mathrm{St}=5$. For either value of $\mathrm{St}$, the results are notably similar to the ODT HST results in Fig. 3(b). Owing to the absence of $\phi$ dependence of $\epsilon$ for the set of ODT HIT cases, the comparison to the current ODT results cannot be considered definitive. Nevertheless, the comparison conveys the impression that ODT exhibits less dependence to the flow configuration than does DNS, subject to the additional caveat that there is not a consistent basis for comparison of the DNS HST and HIT results. The various noted ambiguities are partially alleviated in Sec. IV G by formulating a consistent comparison of ODT results for HIT and HST.

An additional consideration is that $\operatorname{Re}_{\lambda}=70$ for the HIT case, which is higher than for the HST case. ODT is designed to be scale free, and hence to show self-similar behavior, except where this is superseded at large scales by boundary conditions or at small scales by the viscous penalty. As such, it does not capture transitional behavior at moderate $\operatorname{Re}_{\lambda}$. The present focus on the $\operatorname{Re}_{\lambda}=43$ case is motivated by the availability of comparison data rather than optimality of this transitional case for assessment of ODT. The mass loading dependence that is examined is potentially subject to progressive transitional behavior because the TKE is increasingly attenuated as the loading increases. Section IV F shows a comparison of ODT results to the limited DNS data for HST at higher $\operatorname{Re}_{\lambda}$.

Figure 4 shows the effect of mass loading $\phi$ on $k$ and $\epsilon$ normalized by the single-phase values. The degree of agreement is significant given that one parameter was tuned to optimize both quantities at $\phi=0.8$.

Investigating $u_{1}^{\prime 2} / k$ and $u_{2}^{\prime 2} / k$, a limitation of ODT becomes evident (Fig. 5). The ODT results imply a slight reduction of anisotropy as particle loading increases, which is plausible because the two-way coupling provides more degrees of freedom for equilibration of component variances. However, the DNS results indicate that the streamwise variance is insensitive to loading while the variance aligned with the mean shear is strongly affected by the overall TKE attenuation. This suggests some flow behavior that is not readily captured by statistical modeling, perhaps reflecting the transitional $\operatorname{Re}_{\lambda}$ value for this case that was discussed with reference to Fig. 3. As the flow becomes increasingly transitional with increasing $\phi$, the flow structure is potentially subject to qualitative modifications that account for the observed tendency. As noted in Sec. I, this behavior is also seen in turbulent boundary layers, but it is unclear whether this is an effect of the mean shear or the wall.

Notwithstanding these caveats, the available evidence supports the behavior indicated by the DNS results in Fig. 5. Although ODT time advances all three components of the flow field and the particle drag law, the model representation of vortical eddy motions is implemented only in the domain-aligned direction, so the model is incomplete with respect to possible anisotropy induced by two-way interactions. The anisotropy of single-phase ODT that is indicated by Table I reflects a property of model observables rather than a full representation of anisotropic motion. We therefore conclude based on Fig. 5 and the other noted evidence that, at best, ODT captures the fluid response to fluid-particle interactions globally rather than at the level of directional effects. This does not preclude a sound statistical treatment of particle trajectories, which has been demonstrated for other flows and will be addressed for HST in future work.

\section{F. Additional comparisons of ODT and DNS parameter dependences}

In this section, the $\operatorname{Re}_{\lambda}, \mathrm{St}$, and $\phi$ dependences of $k$ and $\epsilon$ are examined for ODT and DNS. The representative cases here are chosen to be $\mathrm{Re}_{\lambda}=43$ and $84, \mathrm{St}=1$ and 5 , and $\phi=0.0-0.8$ based on the available DNS data of Gualtieri et al. [3] and Battista et al. [13]. Figure 6 shows the parameter dependence of $\epsilon$ normalized by the single-phase quantity $\epsilon_{0}$. For both $\operatorname{Re}_{\lambda}$ values, $\epsilon$ is nearly independent of St. ODT simulations for $\operatorname{Re}_{\lambda}>84$ were carried out and show the same 


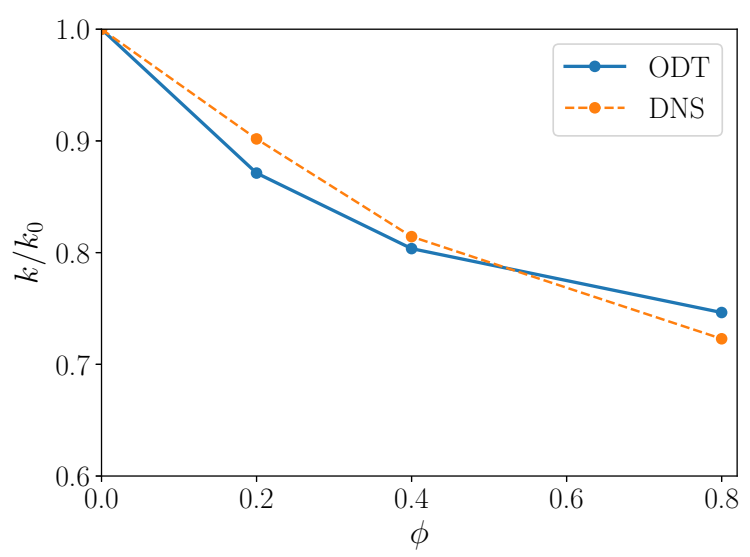

(a)

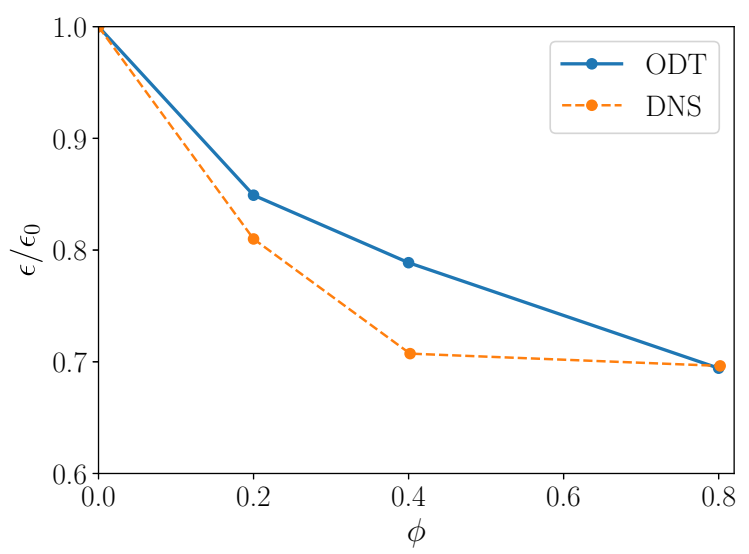

(b)

FIG. 4. Dependence of the fluid-phase TKE (a) and its dissipation rate (b), normalized by the values for the single-phase case $\left(k_{0}\right.$ and $\left.\epsilon_{0}\right)$, on mass loading. (Same cases as in Fig. 3.)

insensitivity. The results show that $\phi$ seems to be the dominant parameter in determining $\epsilon$, which decreases with increasing $\phi$.

Variation of $\phi$ has the same effect on $k / k_{0}$, as shown in Fig. 7. It decreases with increasing $\phi$. However, $\operatorname{Re}_{\lambda}$ and $\mathrm{St}$ seem to have a significant effect as well. Higher St decreases the attenuation of $k$ in ODT and DNS. ODT is tuned to capture the DNS data for $\operatorname{Re}_{\lambda}=43, \mathrm{St}=1$, and $\phi=0.8$, so all other results are interpreted as predictions. While ODT overpredicts the attenuation for $\operatorname{Re}_{\lambda}=84$, the trend for increasing St is captured. Nevertheless, in Battista et al. [13] only a single data point for $\mathrm{St}=5$ is provided and should be interpreted cautiously. It is interesting that ODT predicts stronger St sensitivity at low [Fig. 7(a)] than at high [Fig. 7(b)] $\operatorname{Re}_{\lambda}$. A test of this using DNS or other means would be instructive.

The same St and $\phi$ variations were run for higher $\operatorname{Re}_{\lambda}$ values. The results were not significantly different from the results for $\operatorname{Re}_{\lambda}=84$. Thus, the latter value is in the range of $\operatorname{Re}_{\lambda}$-insensitive behavior, while the $\mathrm{Re}_{\lambda}=43$ ODT results are not fully developed in this sense. Thus, ODT at this $\operatorname{Re}_{\lambda}$ is not representative of fully developed turbulence, and its transitional behavior is not reliably predictive because it is not formulated to capture the phenomenology of this regime. This could impact the comparison to the $\mathrm{Re}_{\lambda}=43$ DNS results, and could additionally impact the comparison 


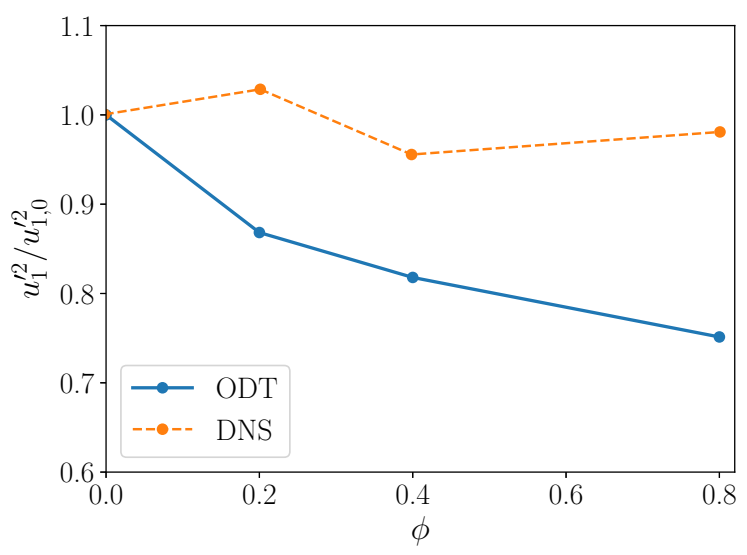

(a)

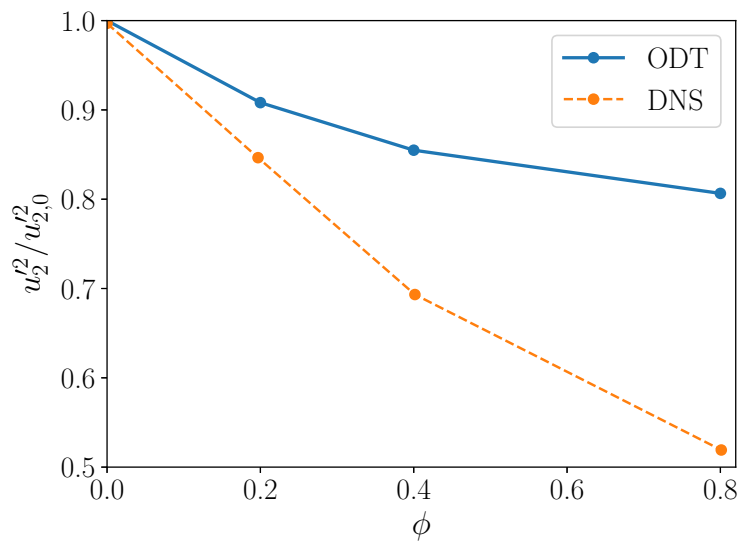

(b)

FIG. 5. Dependence of the velocity variance in (a) streamwise and (b) domain-aligned directions, normalized by the values for the single-phase case $\left(u_{1,0}^{\prime 2}\right.$ and $\left.u_{2,0}^{\prime 2}\right)$, on mass loading. (Same cases as in Fig. 3.)

to the $\operatorname{Re}_{\lambda}=84$ DNS results because $\beta_{p}$ is determined by tuning $\operatorname{Re}_{\lambda}=43$ ODT results. Retuning $\beta_{p}$ to match the $\operatorname{Re}_{\lambda}=84$ DNS case could improve the agreement, but there are no additional data at this or higher $\operatorname{Re}_{\lambda}$ values for validation of the adjusted formulation, so this is not attempted.

A broader concern is that the $\operatorname{Re}_{\lambda}$ values that label the cases refer to unladen flow, but the turbulence intensity decreases with increasing $\phi$. DNS of the two-way-coupling regime has not yet been reported for turbulence levels that are demonstrably fully developed over a significant range of particle loadings. Accordingly, the available DNS data are of limited utility for characterizing the fully developed regime or model validation in that regime. As noted in Sec. IB, scientific understanding and reliable prediction of particle-laden turbulence in the universal subrange of the turbulent cascade would be an important step toward efficient development of SGS closures based on a manageable number of canonical configurations. This point is further elaborated in Sec. IV G.

\section{G. HST versus forced HIT}

An important finding of the HIT study [16] was that comparisons of ODT and DNS particleladen results for which the only change in case specifications was the particle mass loading are not 


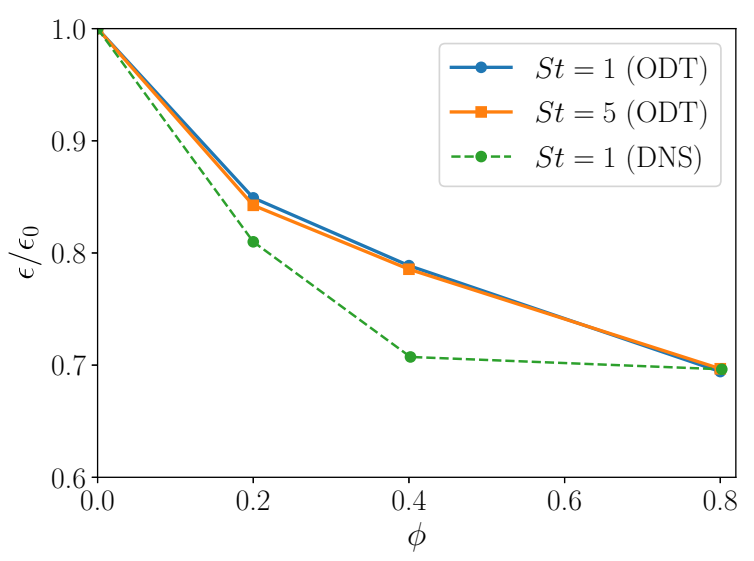

(a) $R e_{\lambda}=43$

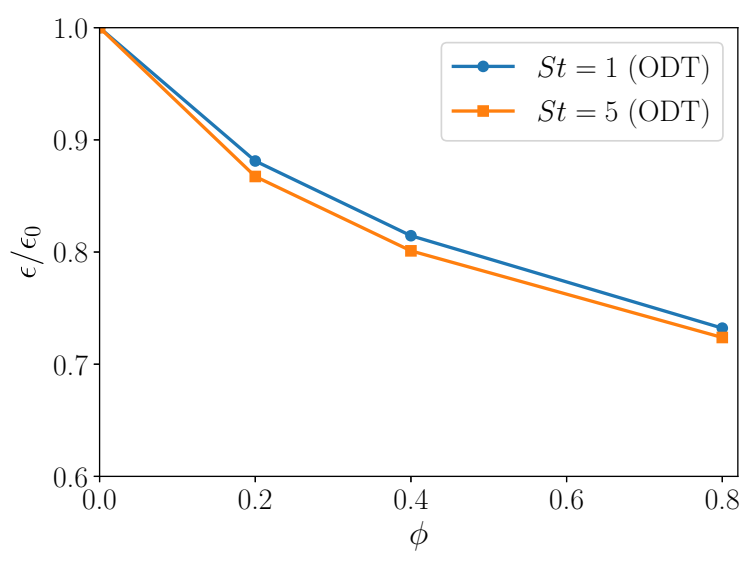

(b) $R e_{\lambda}=84$

FIG. 6. Dependence of the TKE dissipation rate $\epsilon$, normalized by the value for the single-phase case $\left(\epsilon_{0}\right)$, on mass loading for ODT (DNS by Gualtieri et al. [3]).

necessarily consistent. As noted in Sec. IB, this is because a point-particle treatment in two-waycoupled DNS couples the HIT forcing to the particle phase in a way that influences the dissipation rate. Consistent comparisons require case-by-case adjustment of the ODT forcing to match the dissipation rate for the corresponding DNS case. For forced HIT, a convenient feature of two-waycoupled particle-laden ODT is that, in contrast to DNS, the dissipation rate is precisely the value targeted by the assigned forcing rate. This enabled the consistent comparisons of ODT and DNS HIT results in Ref. [16].

Here this approach is used to examine the degree to which ODT results for HST and HIT agree or differ. To compare these flows on a consistent basis, the HIT cases are run with the introduced three-eddy-type formulation. For this purpose, $p_{23}$ is set to $1 / 3$ in Eq. (11) to enforce isotropic energy redistribution, and periodic boundary conditions and the forcing scheme of Ref. [16] are used. To match the HST single-phase TKE, the ODT parameter $C$ [Eq. (13)] requires adjustment to 1.3. Notwithstanding these provisions, the evaluation of $\beta_{p}$ in different ways for the two flows owing to the different types of available DNS output statistics, as discussed in Sec. IV E, precludes full consistency so the comparisons and their interpretations should be considered tentative. 


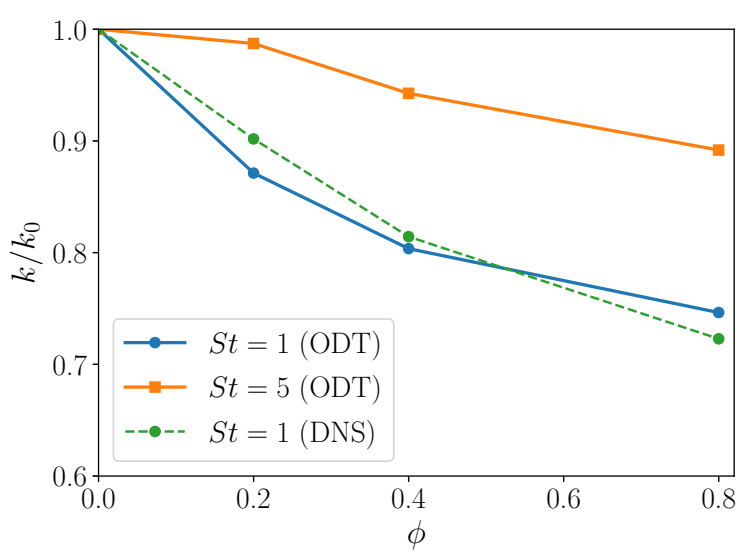

(a) $R e_{\lambda}=43$

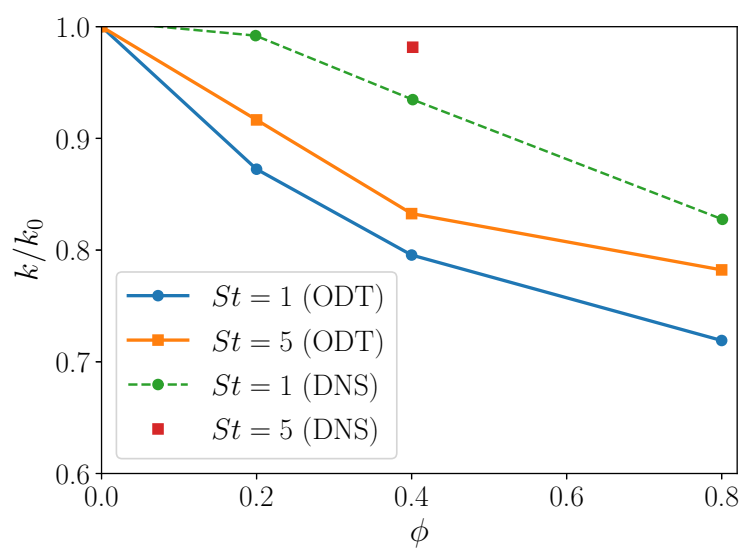

(b) $R e_{\lambda}=84$

FIG. 7. Dependence of the fluid-phase TKE $k$, normalized by the value for the single-phase case $\left(k_{0}\right)$, on mass loading for ODT. (a) $\operatorname{Re}_{\lambda}=43$, (DNS by Gualtieri et al. [3]), (b) $\operatorname{Re}_{\lambda}=84$ (DNS by Battista et al. [13]).

Results of HIT runs for the St and $\phi$ values of the parameter studies in Sec. IV F are shown in Fig. 8, which compares $k / k_{0}$ results for cases in which the dissipation rate is fixed at the single-phase HST value with results for which the dissipation rate for each case is adjusted to the HST value shown in Fig. 6(b). One observation is that the differences between Fig. 8(a), in which the dissipation rate for all cases was set equal to its value for the single-phase case, and Fig. 8(b), in which the dissipation rate for each case was set equal to the HST value for the corresponding mass loading and St value, are significant. Another observation is that the results for the adjusted cases in Fig. 8(b) are substantially closer to the corresponding HST results in Fig. 7(b) than are the results in Fig. 8(a). Nevertheless, there are notable differences between Figs. 7(b) and 8(b), primarily the greater St dependence of the HST cases. The dissipation-rate adjustment procedure lends some degree of confidence that this reflects the different flow configurations rather than an inconsistent basis for comparison.

The differences between Figs. 7(b) and 8(b) can be understood in terms of the universality of the turbulent cascade at small scales. As noted in Sec. IB, high-St particles interact with larger scales of turbulence than low-St particles, and the larger-scale motions are more sensitive to the 


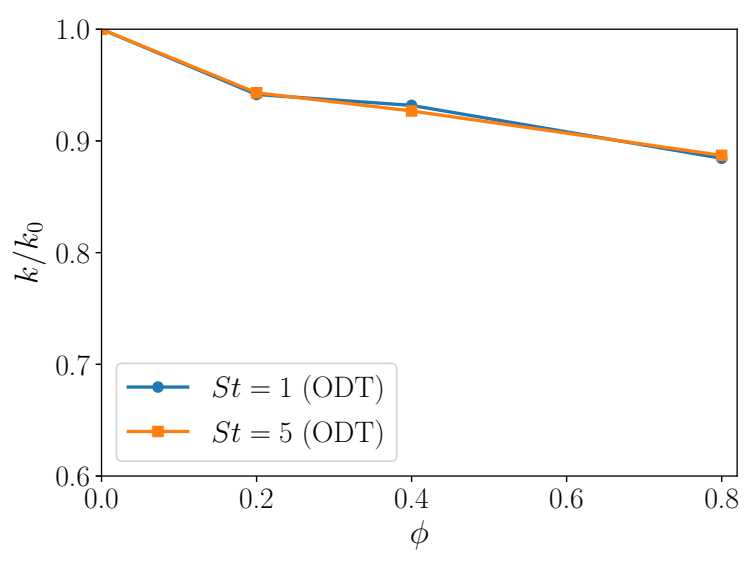

(a) Constant $\epsilon$ set to $\epsilon_{0}$

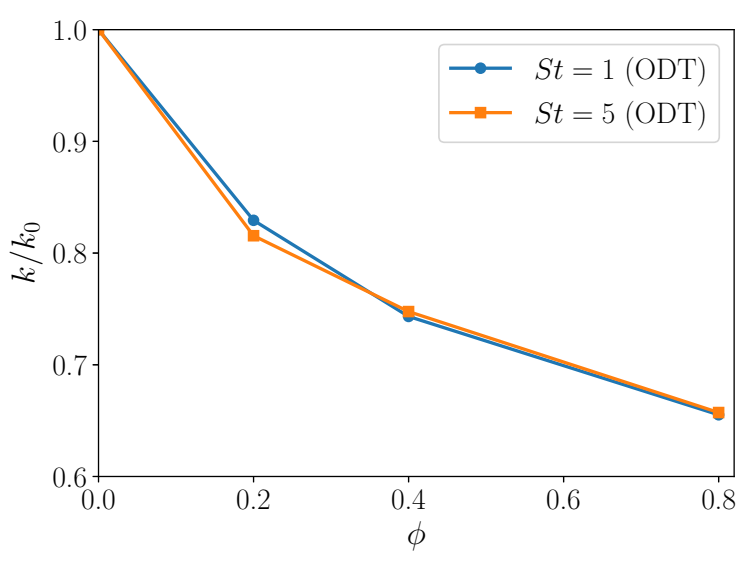

(b) Adjusted $\epsilon(\phi)$ to HST values

FIG. 8. Dependence of the fluid-phase TKE $k$ in forced HIT $\left(\operatorname{Re}_{\lambda}=84\right)$, normalized by the value for the single-phase case $\left(k_{0}\right)$, on mass loading for ODT.

configuration-specific turbulence production and/or decay mechanisms. The stronger sensitivity to the flow configuration for $\mathrm{St}=5$ than for $\mathrm{St}=1$ is consistent with this picture.

These results can be interpreted as indicating that the two flow configurations are marginally approaching universality at $\operatorname{Re}_{\lambda}=84$ in the sense that for low St, corresponding to particle interaction primarily with the smallest flow scales, there is relatively low sensitivity to the flow configuration. This is consistent with the observation in Sec. IV F that HST results are found to be insensitive to $\operatorname{Re}_{\lambda}$ starting at $\operatorname{Re}_{\lambda}=84$, but it also indicates that the latter insensitivity is insufficient to assure universal behavior of the flow over a significant range of St values.

Because ODT is governed by scaling principles, it has a greater tendency than DNS to reach a self-similar state, so universality should be approached more slowly by DNS than by ODT. This suggests that ODT identifies a lower bound on the turbulence intensity required to reach the universal regime of particle-flow interaction by means of DNS or experiments.

Figures 9 and 10 show analogous comparisons involving $k_{p} / k$. Roughly as much $\operatorname{Re}_{\lambda}$ sensitivity is seen in Fig. 9 as in Fig. 7. Figure 10 shows that the dissipation-rate adjustment yields substantially 


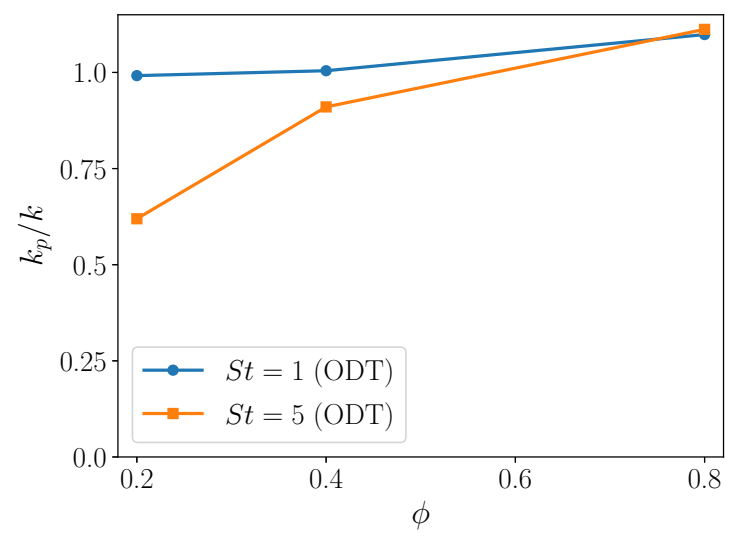

(a) $R e_{\lambda}=43$

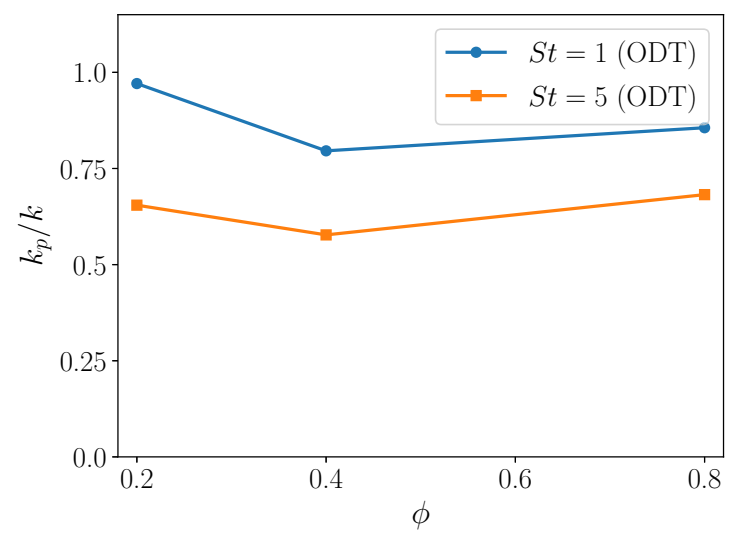

(b) $R e_{\lambda}=84$

FIG. 9. Dependence of the ratio of particle TKE to fluid-phase TKE in HST on mass loading for ODT.

improved agreement with Fig. 9(b). This reinforces the impression that there is not much sensitivity to the flow configuration for $\operatorname{Re}_{\lambda}=84$.

Nonmonotonic dependence of $k_{p} / k$ on $\phi$, consistent with the trends in Fig. 9(b), was also obtained for HIT with $\operatorname{Re}_{\lambda}=62$ (Fig. 6 of Ref. [16]). However, this involved $\phi$-dependent adjustment of the dissipation rate to match a DNS comparison case used in that study. When the $\phi$ excursions were run with the dissipation rate held fixed at the value for $\phi=0$, monotonic trends were obtained (Fig. 11 of Ref. [16]). This underscores the importance of formulating sensitivity studies in a way that minimizes competing influences on the outcomes.

$k_{p} / k$ values are easily extracted from DNS output but have not been reported for the twoway-coupling regime to the authors' knowledge. It is recommended that DNS studies report this fundamental flow property in the future owing to its potential value for model validation purposes and to address the associated conceptual issues that have been identified here. In particular, DNS could test the ODT prediction of nonmonotonic $\phi$ dependence of $k_{p} / k$ for a fixed HST flow configuration, but (as implied by the comparison to ODT HIT results) monotonic dependence if the configuration is adjusted to obtain the same dissipation rate for each $\phi$ value. 


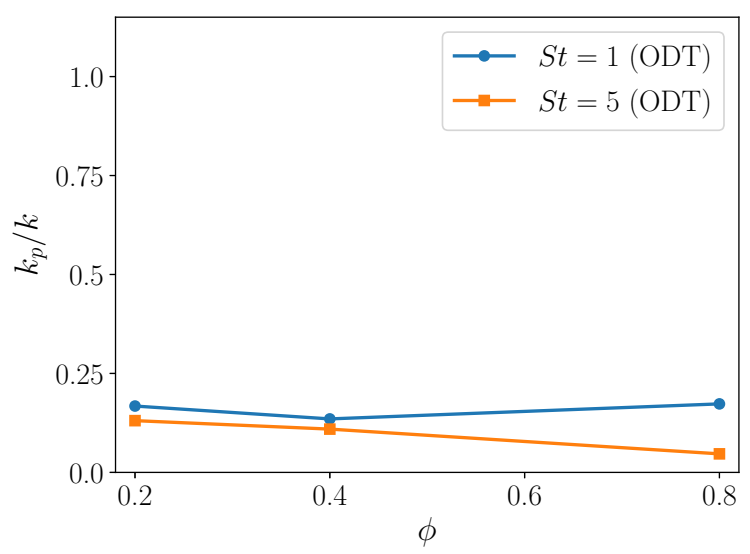

(a) Constant $\epsilon$ set to $\epsilon_{0}$

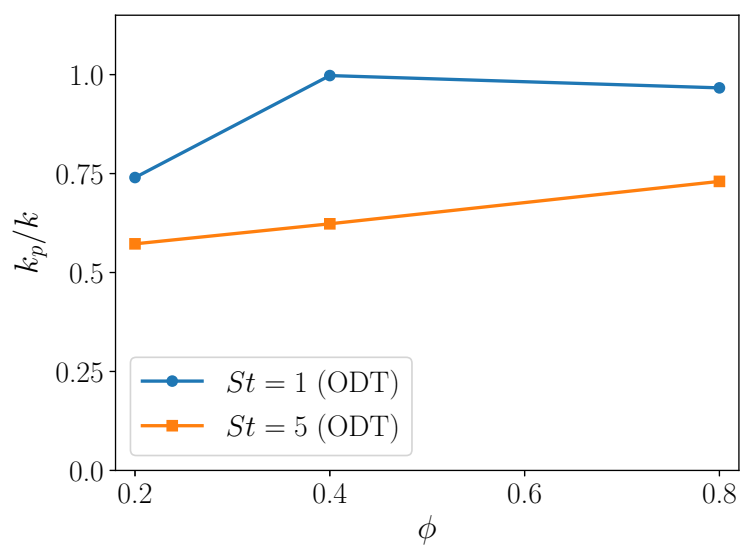

(b) Adjusted $\epsilon(\phi)$ to HST values

FIG. 10. Dependence of the ratio of particle TKE to fluid-phase TKE in $\operatorname{Re}_{\lambda}=84$ forced HIT on mass loading for ODT.

\section{H. One-eddy-type versus three-eddy-type HIT}

To complete the linkage between this study and Ref. [16], the fixed- $\epsilon$ three-eddy-type HIT cases shown in Sec. IV G were rerun with the HIT formulation in Ref. [16]. The results are shown in Fig. 11. Comparison of Fig. 11(a) with Figs 8(a) and Fig. 11(b) with Fig. 10(a) shows that the differences are negligible.

Assuming that further testing for other configurations, not limited to particle-laden flows, further confirms this equivalence, then the three-eddy-type formulation can be considered to be backward compatible with previous ODT formulations while allowing greater flexibility in capturing fluidphase anisotropy. Figure 5 indicates that this has no apparent benefit with regard to capturing the effect of fluid-particle interactions on fluid-phase anisotropy. Nevertheless, it was useful to establish this within a framework that reproduces the single-phase anisotropic structure of HST so that the cause of this deficiency of the model could be clearly identified. 


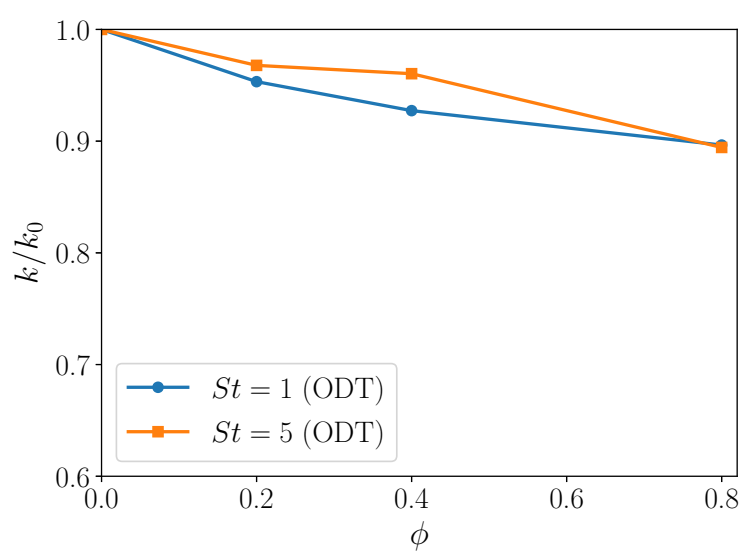

(a) $k / k_{0}$

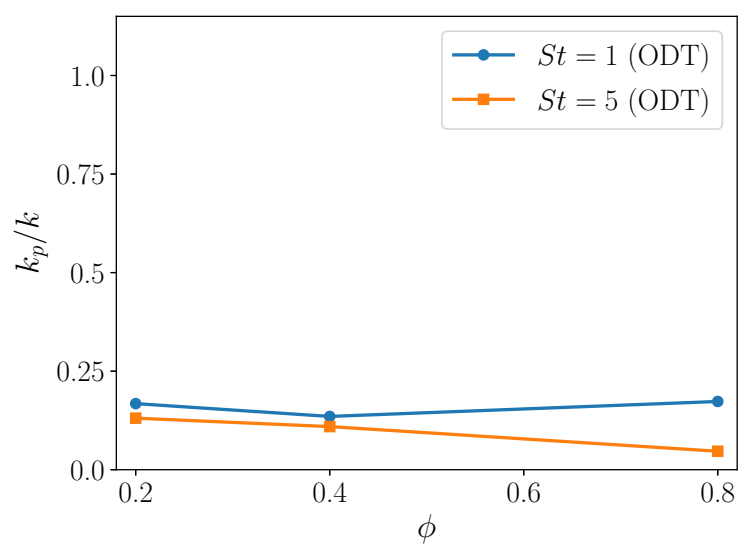

(b) $k_{p} / k$

FIG. 11. Dependence of fluid and particle TKE in $\operatorname{Re}_{\lambda}=84$ forced HIT on mass loading for ODT using the one-eddy-type formulation. Here $\epsilon$ is set to $\epsilon_{0}$.

\section{DISCUSSION}

The present study of particle-laden homogeneous shear turbulence (HST) is an extension of a previous [16] ODT study of turbulence modulation by particles in forced, statistically stationary homogeneous isotropic turbulence (HIT). The key findings and their implications are as follows.

For HST, the present model formulation cannot reproduce the known increase of turbulence anisotropy with increasing particle loading owing to the restriction of the computational domain to a one-dimensional line of sight, but the parameter dependences of global quantities that are not direction dependent are reproduced qualitatively. A consistently normalized comparison with ODT results for particle-laden HIT indicates significant differences between the two flows, but the broader picture is that the parameter dependences and other behaviors are analogous. In particular, the results provide indications that the highest $\mathrm{Re}_{\lambda}$ for which DNS of the two-way-coupled regime is available might be marginally sufficient to capture universal-range behavior, as defined in Sec. IB, for relatively low St and mass loading.

It is shown in Ref. [16] that consistent comparison is essential in order to obtain intuitive, interpretable trends. For present purposes this requires that each HIT case is run with an imposed 
dissipation rate equal to the dissipation rate of the corresponding HST case, for which the dissipation rate is an output rather than an input. Comparisons based on matching the specifications of the single-phase baseline cases are found to exhibit greater, more arbitrary differences between the two flow configurations than consistent comparisons that are based on matching the specifications of each pair of particle-laden cases that are compared.

To the authors' knowledge, this is the first instance of a consistent case-by-case comparison of turbulence modulation effects in two distinct flow configurations. Consistent comparisons between DNS results for different flow configurations are difficult for reasons explained in Sec. I B.

Additionally, the paucity of comparison data here and in Ref. [16] may reflect the high computational cost of DNS of turbulent particle-laden flow. This underscores the importance of extracting as much useful information as possible from DNS cases. DNS studies are generally not focused on model validation, so it is incumbent on modelers to provide guidance concerning basic steps that would enhance the impact of DNS results in this regard.

For two-way-coupled particle-laden turbulence, several examples are noted. The ODT results in Secs. IV G and IV H include parameter studies with respect to the ratio of particle to fluid kinetic energy. This fundamental property of multiphase flow is not typically reported in DNS studies, so greater regularity in this regard is recommended. In particular, DNS could test the ODT prediction of nonmonotonic dependence of $k_{p} / k$ on mass loading for a fixed HST flow configuration, whose plausibility is supported by a cross-comparison of ODT HST and HIT results.

Additionally, basic metrics of the particle-fluid interaction such as the mean (where nonzero) and variance of the slip velocity (broken out by component for anisotropic cases, and reported at multiple locations for inhomogeneous cases) could contribute to the advancement of multiphase turbulent flow modeling. In particular, slip-velocity statistics, even if only mean $\operatorname{Re}_{p}$ values, would be a more robust basis for evaluating the ODT model parameter $\beta_{p}$ than is presently available.

For anisotropic flows such as HST, ODT has been extended here by introducing three eddy types instead of one, where a given eddy type transfers kinetic energy between two of the three velocity components, in this manner emulating a two-dimensional eddy motion. (The one-eddy-type formulation redistributes kinetic energy among all three components during an eddy event.) This introduces an additional degree of freedom that enables quantitatively accurate representation of the full three-dimensional anisotropic structure of single-phase HST during its self-similar regime of transient development. The extension includes the option of isotropic coupling of velocity components, albeit two at a time, as a special case, so it is applicable to any flow to which ODT as originally formulated can be applied. It is recommended as a general-purpose alternative to that formulation because it subsumes all of its capabilities and adds to them.

As noted, this extension is not sufficient to capture the increase of turbulence anisotropy with increasing particle loading, but its physically reasonable global parameter dependences suggest that it might provide useful guidance for the development of parameterized subgrid-scale (SGS) closure of large-eddy simulations (LESs) of turbulence modulation by particles. HST in a finite domain reaches a statistically stationary state with a unique dependence of the energy dissipation on the imposed shear and the particle physical properties and number density. This and other reasons for continued focus on this flow configuration, in tandem with DNS studies that provide the needed inputs identified above, have been noted in Sec. IB. The availability of suitable DNS inputs will be a consideration affecting the choice of ODT flow configuration in the future.

The turbulent boundary layer is a distinct and important flow that likewise requires ongoing attention, focusing on the specific requirements for near-wall SGS closure of particle-laden flow. The lateral inhomogeneity of boundary layers introduces turbophoresis. A natural first step is to investigate turbophoresis in a laterally inhomogeneous unbounded periodic flow, as in Ref. [35], which identified phenomenology using a continuum two-way-coupled model of the particle phase. Comparison to two-way-coupled particle-laden ODT simulation of that flow will be of interest.

The one-way-coupled boundary layer was the first application of particle-laden ODT [24]. The present two-way-coupled treatment will be incorporated into the cylindrical formulation of 
ODT [21] for comparison to recent two-way-coupled pipe-flow DNS results [36]. Collectively, these research directions will enable needed parameter studies in support of SGS closure for LES application to particle-modulated turbulence.

An important feature of ODT is that all flow processes evolve with full spatial and temporal resolution, allowing multiple coupled microphysical subprocesses to be incorporated without much loss of fidelity beyond the inherent model limitations, as documented in the cited references and elsewhere. For particle-laden HST, multiphysics environments that will be investigated include particle coupling to a variable-temperature flow, involving addition of thermophoretic forcing to the drag law as well as droplet evaporation impacting the temperature field through latent-heat as well as sensible-heat effects, with feedback to the flow owing to temperature-dependent viscosity. The capability to affordably explore the parameter space spanned by $\mathrm{Re}_{\lambda}$, St, density ratio, particle number density, and parameters characterizing the thermal couplings is not otherwise available.

\section{ACKNOWLEDGMENT}

This project has received funding from the European Union Horizon-2020 Research and Innovation Program Grant Agreement No 675676.

[1] M. Tanaka and D. Teramoto, Modulation of homogeneous shear turbulence laden with finite-size particles, J. Turb. 16, 979 (2015).

[2] R. Monchaux and A. Dejoan, Settling velocity and preferential concentration of heavy particles under two-way coupling effects in homogeneous turbulence, Phys. Rev. Fluids 2, 104302 (2017).

[3] P. Gualtieri, F. Picano, G. Sardina, and C. M. Casciola, Clustering and turbulence modulation in particleladen shear flows, J. Fluid Mech. 715, 134 (2013).

[4] A. H. Abdelsamie and C. Lee, Decaying versus stationary turbulence in particle-laden isotropic turbulence: Heavy particle statistics modifications, Phys. Fluids 25, 033303 (2013).

[5] G. Hetsroni, Particle-turbulence interaction, Int. J. Multiphase Flow 15, 735 (1989).

[6] E. Achenbach, Vortex shedding from spheres, J. Fluid Mech. 62, 209 (1974).

[7] Y. Tsuji and Y. Morikawa, LDV measurements of an air-solid two-phase flow in a horizontal pipe, J. Fluid Mech. 120, 385 (1982).

[8] G. Hetsroni and M. Solokov, Distribution of mass, velocity and intensity of turbulence in a two-phase turbulent jet, Trans. ASME J. Appl. Mech. 38, 315 (1971).

[9] R. Gore and C. T. Crowe, Effects of particle size on modulating turbulent intensity, Int. J. Multiphase Flow 15, 279 (1989).

[10] K. D. Squires and J. K. Eaton, Particle response and turbulence modification in isotropic turbulence, Phys. Fluids A 2, 1191 (1990).

[11] M. Boivin, O. Simonin, and K. D. Squires, Direct numerical simulation of turbulence modulation by particles in isotropic turbulence, J. Fluid Mech. 375, 235 (1998).

[12] A. Ferrante and S. Elghobashi, On the physical mechanisms of two-way coupling in particle-laden isotropic turbulence, Phys. Fluids 15, 315 (2003).

[13] F. Battista, P. Gualtieri, J.-P. Mollicone, and C. M. Casciola, Application of the Exact Regularized Point Particle method (ERPP) to particle laden turbulent shear flows in the two-way coupling regime, Int. J. Multiphase Flow 101, 113 (2018).

[14] D. Richter, Turbulence modification by inertial particles and its influence on the spectral energy budget in planar Couette flow, Phys. Fluids 27, 063304 (2015).

[15] M. Righetti and G. Romano, Particle-fluid interactions in a plane near-wall turbulent flow, J. Fluid Mech. 505, 93 (2004). 
[16] M. Fistler, A. Kerstein, D. O. Lignell, and M. Oevermann, Turbulence modulation in particle-laden, stationary homogeneous isotropic turbulence using one-dimensional-turbulence, Phys. Rev. Fluids 5, 044308 (2020).

[17] A. R. Kerstein, One-dimensional turbulence: Model formulation and application to homogeneous turbulence, shear flows, and buoyant stratified flows, J. Fluid Mech. 392, 277 (1999).

[18] A. R. Kerstein, W. T. Ashurst, S. Wunsch, and V. Nilsen, One-dimensional turbulence: Vector formulation and application to free shear flows, J. Fluid Mech. 447, 85 (2001).

[19] W. T. Ashurst and A. R. Kerstein, One-dimensional turbulence: Variable density formulation and application to mixing layers, Phys. Fluids 17, 025107 (2005).

[20] D. Lignell, A. Kerstein, G. Sun, and E. Monson, Mesh adaption for efficient multiscale implementation of one-dimensional turbulence, Theor. Comput. Fluid Dyn. 27, 273 (2013).

[21] D. O. Lignell, V. B. Lansinger, J. Medina, M. Klein, A. Kerstein, H. Schmidt, M. Fistler, and M. Oevermann, One-dimensional turbulence modeling for cylindrical and spherical flows: Model formulation and application, Theor. Comput. Fluid Dyn. 32, 495 (2018).

[22] A. Movaghar, M. Linne, M. Oevermann, F. Meiselbach, H. Schmidt, and A. R. Kerstein, Numerical investigation of turbulent-jet primary breakup using one-dimensional turbulence, Int. J. Multiphase Flow 89, 241 (2017).

[23] A. Movaghar, M. Linne, M. Herrmann, A. Kerstein, and M. Oevermann, Modeling and numerical study of primary breakup under diesel conditions, Int. J. Multiphase Flow 98, 110 (2018).

[24] J. R. Schmidt, J. O. L. Wendt, and A. R. Kerstein, Non-equilibrium wall deposition of inertial particles in turbulent flow, J. Stat. Phys. 137, 233 (2009).

[25] G. Sun, D. O. Lignell, J. C. Hewson, and R. G. Craig, Particle dispersion in homogeneous turbulence using the one-dimensional turbulence model, Phys. Fluids 26, 103301 (2014).

[26] G. Sun, J. C. Hewson, and D. O. Lignell, Evaluation of stochastic particle dispersion modeling in turbulent round jets, Int. J. Multiphase Flow 89, 108 (2017).

[27] P. Gualtieri, F. Battista, and C. M. Casciola, Turbulence modulation in heavy-loaded suspensions of tiny particles, Phys. Rev. Fluids 2, 034304 (2017).

[28] P. Bradshaw and W. A. Woods, An Introduction to Turbulence and Its Measurement (Pergamon Press, New York, 1971).

[29] M. M. Rogers and P. Moin, The structure of the vorticity field in homogeneous turbulent flows, J. Fluid Mech. 176, 33 (1987).

[30] R. Clift, J. R. Grace, and M. E. Weber, Bubbles, Drops and Particles (Academic Press, Cambridge, 1978).

[31] S. Elghobashi and G. C. Truesdell, Direct simulation of particle dispersion in a decaying isotropic turbulence, J. Fluid Mech. 242, 655 (1992).

[32] L. Schiller and A. Naumann, Über die grundlegenden Berechnungen bei der Schwerkraftaufbereitung, Z. Ver. Deut. Ing. 77, 318 (1933).

[33] S. Elghobashi, On predicting particle-laden turbulent flows, Appl. Sci. Res. 52, 309 (1994).

[34] V. Pandey, P. Perlekar, and D. Mitra, Clustering and energy spectra in two-dimensional dusty gas turbulence, Phys. Rev. E 100, 013114 (2019).

[35] A. Sozza, M. Cencini, S. Musacchio, and G. Boffetta, Drag enhancement in a dusty Kolmogorov flow, Phys. Rev. Fluids 5, 094302 (2020).

[36] F. Battista, J.-P. Mollicone, P. Gualtieri, R. Messina, and C. M. Casciola, Exact regularized point particle (ERPP) method for particle-laden wall-bounded flows in the two-way coupling regime, J. Fluid Mech. 878, 420 (2019). 